\title{
COMPLETENESS AND COMPACTNESS IN LINEAR TOPOLOGICAL SPACES $\left({ }^{(}\right)$
}

\author{
BY \\ HERON SHERWOOD COLLINS
}

1. Introduction. The object of this paper is a study of the equicontinuous $\mathrm{w}^{*}\left(\mathrm{e}-\mathrm{w}^{*}\right)$ topology, a generalization of the bounded-weak-* topology [ 1 ; $22 ; 5]$ for the adjoint of a real or complex 1.t.s. (linear topological space), together with an examination of weak compactness and of two properties for l.t.s.'s (full and pseudo-completeness) which are closely related to completeness and are most naturally stated in terms of the e-w* topology. It is shown here that if $(X, \mathcal{T})$ is an 1.t.s. and if $\mathcal{T}_{c}$ denotes the locally convex topology for $X$ having a local base (base for neighborhoods of 0 ) consisting of all convex $\mathcal{\tau}$ neighborhoods of 0 , then $\left(X, \mathcal{T}_{c}\right)$ is complete if and only' if $(X, \mathcal{T})$ is pseudo-complete. In addition, although pseudo-completeness is weaker than full completeness in arbitrary l.t.s.'s, it is shown that when $(X, \mathcal{T})$ is pseudo-metrisable full completeness and pseudo-completeness for $(X, \mathcal{T})$ coincide, both being equivalent to the requirement that $\left(X, \mathcal{T}_{c}\right)$ be complete. These results on pseudo-completeness and full completeness represent the author's attempt to name and study more thoroughly two properties for l.t.s.'s which have appeared frequently in the literature (e.g., see [3, pp. 131-132], [1, Theorem 1.4], [19, Theorem 5], [15, Theorem 8.10]).

The main problem concerning the e- $\mathrm{w}^{*}$ topology and related topologies which concerns us in this paper is the following: if $X$ is an 1.t.s., when is it true that the following three topologies for the adjoint space $X^{*}$ of continuous linear functionals coincide: the finest topology (distinguish between topology and 1.t.s. topology!) coinciding with the $\mathrm{w}^{*}$ (=weak-*) topology on each equicontinuous subset of $X^{*}$, the finest locally convex 1.t.s. topology coinciding with $\mathrm{w}^{*}$ on equicontinuous sets, and the finest uniform convergence topology (defined by the polars in $X^{*}$ of certain subsets of $X$ ) coinciding with w* on equicontinuous sets? A sufficient condition that these three topologies coincide is that $X$ be pseudo-metrisable. Additional results concerning these and related topologies are given in Part 2, and corollaries of these results are drawn which duplicate or generalize previous work of Dieudonné and Schwartz [6, p. 35 and Theorem 5], Krein and Šmulian [19, Theorems 5 and 6], Alaoglu [1, Theorem 1.4], and Katětov [15, Theorem 8.10].

Presented to the Society, October 25, 1952; received by the editors October 16, 1952 and, in revised form July $19,1954$.

(1) This work was done at Tulane University under contract N 7-ONR-434, Task Order 111, Navy Department, the Office of Naval Research, U.S.A., and represents the major part of a thesis submitted to Tulane University July, 1952, in partial fulfillment of the requirements for the Ph.D. degree. The author wishes to express his thanks to Professor B. J. Pettis for his many helpful suggestions. 
One of our main results is the theorem that when $X$ is an 1.t.s. (neither local convexity nor separation is assumed) and $X$ with the Mackey topology ([20] and [6]) is pseudo-complete, then in $X$ all of the various weak compactness notions (excluding weak sequential compactness) coincide. Included among these equivalences are several types of weak compactness (in particular, a notion studied in $[26 ; 27]$ ) not considered in the later papers on weak compactness, and our work includes work by Šmulian $[26 ; 27]$, Phillips [23], Eberlein [8], Dieudonné and Schwartz [6], and by Grothendieck [11].

Definitions AND NOTATION. Throughout this paper we shall be concerned with real linear topological spaces $X$, although our results can be obtained for complex spaces by replacing in the proofs of the various theorems the word "symmetric" by "circled" (if $X$ is an 1.t.s. over a normed field of scalars, $A \subset X$ is symmetric [circled] if $x \in A$ implies $-x \in A$ [if $a x \in A$ for every $x \in A$ and every scalar $a$ such that $|a| \leqq 1]$ ). The neutral element of $X$ will always be denoted by 0 . A topology $\mathcal{\tau}$ for a linear space $X$ is called linear if addition and scalar multiplication are both continuous functions on the appropriate product spaces into $X$. A base for neighborhoods of 0 is always called a local base, and $X$ is pseudo-metrisable if $X$ has a countable local base. The notation $\{x$ :-(proposition about $x)-\}$ is, by definition, the set of all points $x$ such that the proposition about $x$ is correct. Finally, for $E \subset X$ and $F \subset X^{*}, E^{0}$ and $F_{0}$ denote respectively the polar of $E$ in $X^{*}$ and $F$ in $X$.

When $X$ is an 1.t.s. the $e-w^{*} \operatorname{topolog} y$ for $X^{*}$ is defined as the finest (not necessarily linear) topology which coincides with the $\mathrm{w}^{*}$ topology on equicontinuous sets, and $X$ is pseudo-complete [fully complete] if the e-w* and $\mathrm{w}^{*}$ closures for maximal proper [arbitrary] linear subspaces of $X^{*}$ coincide.

Our results can be divided roughly into three parts: Part 1 gives some preliminary facts concerning the e- $\mathrm{w}^{*}$ topology and pseudo-completeness but is devoted primarily to weak compactness; Part 2 contains a discussion of a general method of defining topologies in 1.t.s.'s, with special emphasis on the e-w* topology; the final Part 3 discusses fully complete spaces.

\section{Part 1. Pseudo-completeness and Weak compactness}

2. Preliminaries. For purposes of reference we shall list at the outset those compactness conditions (and their definitions) with which we shall be concerned throughout Part 1 . All of the types of compactness listed here with the exception of (7) and (8) below can be stated in more general context than that of the weak topology of an 1.t.s., of course, but we restrict ourselves to these except for a few remarks following these listings.

Consider then, these conditions on a subset $M$ of an 1.t.s. $X:$ (1) the weak closure of $M$ is weakly compact (=bicompact); (2) the weak closure of $M$ is weakly Fréchet compact (every infinite subset of $M$ has a weak accumulation point in $M$ ); (3) the weak closure of $M$ is weakly cluster point compact (every sequence in $M$ has a weak cluster point in $M$, where $x \in X$ is a weak cluster 
point of the sequence $\left\{x_{n}\right\}$ if given any weak neighborhood $G$ of $x$ and any integer $k$ there is an integer $n>k$ such that $\left.x_{n} \in G\right)$; (4) $M$ is weakly Fréchet compact in $X$ (every infinite subset of $M$ has a weak accumulation point somewhere in $X) ;(5) M$ is weakly cluster point compact in $X$ (every sequence in $M$ has a weak cluster point in $X) ;(6) M$ is weakly semi-cluster point compact in $X$ (every sequence in $M$ has a weak semi-cluster point in $X$, where $x \in X$ is a weak semi-cluster point of $\left\{x_{n}\right\}$ if given any weak subbasis neighborhood $G$ of $x$ and any integer $k$ there is an integer $n>k$ such that $\left.x_{n} \in G\right)$; (7) given any sequence $\left\{x_{n}\right\}$ in $M$ there' exists a point $x$ in $X$ such that $f \in X^{*}$ implies there is a subsequence $\left\{x_{n_{i}}\right\}$ of $\left\{x_{n}\right\}$ such that $\left\{f\left(x_{n_{i}}\right)\right\}$ converges to $f(x) ;(8) M$ is mean compact (given any sequence $\left\{x_{n}\right\}$ in $M$ there is a point $x$ in $X$ such that $\left.f \in X^{*} \operatorname{implies}_{\lim _{n}} \inf f\left(x_{n}\right) \leqq f(x) \leqq \lim _{n} \sup f\left(x_{n}\right)\right)$; and (9) $M$ is weakly sequentially compact in $X$ (every sequence chosen from $M$ has a subsequence converging weakly to some point of $X$ ).

Some of the relationships between the above conditions are quite obvious. For example, when $X$ is an arbitrary topological space and the modifiers "weak" and "weakly" are replaced by " $\mathcal{T}$," where $\mathcal{T}$ is the topology of $X$, it is clear that $(1) \rightarrow(2) \leftrightarrow(3) \rightarrow(4) \rightarrow(5) \rightarrow(6)$ and (9) $\rightarrow(4)$ (a more detailed discussion of some of these relationships under general topological conditions can be found in [11]). In addition, when $X$ is an 1.t.s. and $\mathcal{T}$ is the weak topology of $X$ (i.e., the situation in (1) through (9) above; this case concerns us henceforth) it is easily seen that (6) and (7) are equivalent, both implying (8). The remainder of the implications above are not quite so obvious. In 1938 Smulian [26] proved that (8) and (9) were equivalent for $X$ a Banach space and several years later he showed [27, Theorem 5] that, also for Banach spaces, (4), (8), and (9) were equivalent. Phillips repeated the first Smulian result somewhat later [23, Theorem 1.6], using different methods. Eberlein made more progress in $[8$, p. 53$]$ by showing the equivalence of (1) and (4) for Banach spaces. In their 1950 paper [6, pp. 88-89] Dieudonné and Schwartz proved that (1), (4), and (9) were the same in $F$ and $L F$ spaces. The final result to date is found in Grothendieck's work [11, p. 177] where it is shown that (1) and (5) coincide in complete (actually complete only with respect to the Mackey topology) locally convex Hausdorff spaces. Concerning the latter theorem, we should remark that our main compactness theorem (Corollary 3.3) and Grothendieck's theorem lean heavily on rephrasings of a lemma due to Eberlein [8, p. 52].

The principal purpose of Part 1 is to present a result (Corollary 3.3) which gives a sufficient condition on any l.t.s. that conditions (1) through (8) be equivalent. So far as we know this result includes all previous work on this subject. The derivation we give here is considerably more direct than that of Grothendieck although not as informative from a general topological standpoint.

For this theorem we shall need the notion of the e-w* topology, which has 
been discussed for Banach spaces by Alaoglu [1, Theorem 1.4], Munroe [22], and Dieudonné [5]; and for $F$ spaces by Dieudonné and Schwartz $[6$, p. 84].

3. The e-w* topology and pseudo-completeness. Recall that when $X$ is an 1.t.s., the e-w* topology is defined as the finest topology for the adjoint $X^{*}$ which coincides with the $\mathrm{w}^{*}$ topology on each equicontinuous set. This topology can be described more explicitly by either of the following descriptions of its open sets or its closed sets: $H \subset X^{*}$ is e-w* open [e-w* closed] if and only if, for each equicontinuous set $S$ in $X^{*}, H \cap S$ is open [closed] in the relative $\mathrm{w}^{*}$ topology of $S$. If we denote by $S(X)$ the family of all w* closed, convex, symmetric and equicontinuous subsets of $X^{*}$ (or, equivalently, the collection of all polars in $X^{*}$ of neighborhoods of 0 in $X$ ) another less obvious description is the following: $H$ is e-w* open [F is e-w* closed] if and only if $H \cap S$ is open in the relative $\mathrm{w}^{*}$ topology of $S\left[F \cap S\right.$ is $\mathrm{w}^{*}$ compact ], for every $S \in S(X)$. It is clear that if $H$ is e-w* open then this openness condition holds, since each $S \in S(X)$ is equicontinuous. Conversely, suppose $H \cap S$ is open in the $\mathrm{w}^{*}$ relative topology of $S$, for every $S \in S(X)$. Then, if $A \subset X^{*}$ is equicontinuous, there exists an $S \in S(X)$ such that $A \subset S$; hence $H \cap A=H \cap A \cap S=H \cap S \cap A$. By hypothesis, there exists a w* open set $G$ such that $H \cap S=G \cap S$; therefore $H \cap A=H \cap S \cap A=G \cap S \cap A=G \cap A$, the latter since $A \subset S$. Thus, $H$ is e-w* open. The dual statement follows as easily: if $F$ is e-w* closed, then $S \in S(X)$ implies $F \cap S$ is closed in the w* relative of $S$. But $S$ is w* compact; hence $F \cap S$ is w* compact. Conversely, if $F \cap S$ is w* compact for every $S \in S(X)$, fix $A \subset X^{*}$ equicontinuous. As before, $A \subset S$ for some $S \in S(X)$ and therefore $F \cap A=F \cap A \cap S=F \cap S \cap A$. Since $F \cap S$ is w* compact (hence w* closed), there exists $\mathrm{w}^{*}$ closed $C$ such that $F \cap S=C \cap S$. But then $F \cap A=C \cap S \cap A=C \cap A$, and $F \cap A$ is closed in the w* relative topology of $A$.

Recall that the 1.t.s. $X$ is pseudo-complete if the $\mathrm{w}^{*}$ and $\mathrm{e}-\mathrm{w}^{*}$ closures for maximal proper linear subspaces of $X^{*}$ coincide. An entirely equivalent formulation is given by the following statement: every (nonzero) linear functional (real valued, additive, and homogeneous function) on $X^{*}$ whose null space is $e-w^{*}$ closed also has its null space $w^{*}$ closed. The relationship between pseudo-completeness and completeness (the latter is used in the sense that every cauchy net [16] (or filter) converges to some point of $X$ ) is discussed in Theorems 1 and 2, and in more detail in Part 3.

Lemma 1. Let $X$ be an l.t.s. and $K$ be a convex subset of $X$. If $f$ is a linear functional on $X$ and if $f^{-1}(b) \cap K$ is closed in the relative topology of $K$ for every real number $b$, then $f$ is continuous on $K$ (the restriction of $f$ to $K$ is continuous in the relative topology of $K$ ).

Proof. If $f$ were not continuous on $K$ there would exist a net $\left\{x_{a}: a \in A\right\}$ in $K$ converging to a point $x$ of $K$ such that $\left\{f\left(x_{a}\right): a \in A\right\}$ does not converge 
to $f(x)$. We may extract from $\left\{x_{a}: a \in A\right\}$ either a subnet $\left\{x_{b}: b \in B\right\}$ such that $f\left(x_{b}\right)-f(x)>\epsilon$, all $b \in B$, or a subnet $\left\{x_{c}: c \in C\right\}$ such that $f\left(x_{c}\right)-f(x)<-\epsilon$, all $c \in C$, where $\epsilon>0$. We can thus assume that $f\left(x_{a}\right)-f(x)>\epsilon$, all $a \in A$. Let $\left\{y_{a}: a \in A\right\}$ be a net constructed from $\left\{x_{a}: a \in A\right\}$ by choosing each $y_{a}$ to be a suitable point on the line segment joining $x_{a}$ and $x$ and $f\left(y_{a}\right)$ to be, for all $a \in A$, a constant $b$ different from $f(x)$. Then $\left\{y_{a}: a \in A\right\}$ converges to $x$, hence $x \in f^{-1}(b) \cap K$. But then $f(x)=b$, a contradiction.

Leмma 2. If $X$ is an l.t.s. and $\phi$ is a linear functional on $X^{*}$, these are equivalent for $\phi:(1) \phi$ is $w^{*}$ continuous on each $S \in S(X)$; (2) $\phi$ is e-w* continuous; and (3) $K_{\phi}$, the null space of $\phi$, is $e$-w* closed.

Proof. If $\phi$ is w $^{*}$ continuous on each $S \in S(X)$, then $G$ open in the reals implies $\phi^{-1}(G) \cap S$ is open in the $\mathrm{w}^{*}$ relative topology of $S$, for every $S \in S(X)$, and thus $\phi^{-1}(G)$ is e-w* open. Thus (1) implies (2). To complete the lemma we prove only that (3) implies (1) since (2) obviously implies (3). To show the continuity of $\phi$ on each $S \in S(X)$ it is sufficient by Lemma 1 to show that $\phi^{-1}(b) \cap S$ is $\mathrm{w}^{*}$ closed in $S$ for every real $b$. Note that $\phi^{-1}(b) \cap S$ $=\left[K_{\phi} \cap\left(S-f_{0}\right)\right]+f_{0}$, for any $f_{0} \in \phi^{-1}(b)$, and since $K_{\phi}$ is e-w* closed and $S-f_{0}$ is equicontinuous, we have $K_{\phi} \cap\left(S-f_{0}\right)$ is w* closed in $S-f_{0}$. But then, by the continuity properties of the $\mathrm{w}^{*}$ topology, $\left[K_{\phi} \cap\left(S-f_{0}\right)\right]+f_{0}$ is $\mathrm{w}^{*}$ closed in $S$. This completes the proof.

If $\left(X^{*}, \mathrm{e}-\mathrm{w}^{*}\right)^{*}$ is used to denote the adjoint space of $\mathrm{e}-\mathrm{w}^{*}$ continuous linear functionals on $X^{*}$, Lemma 2 can be used to establish a result concerning pseudo-completeness which helps clarify the relationship (later stated explicitly in Theorem 2) between pseudo-completeness and completeness for $X$. Clearly $X$ is pseudo-complete if every linear functional $\phi$ on $X^{*}$ whose null space $K_{\phi}$ is e-w* closed also has $K_{\phi} \mathrm{w}^{*}$ closed. By Lemma 2 , then, $X$ is pseudo-complete if and only if $\left(X^{*}, \mathrm{e}-\mathrm{w}^{*}\right)^{*}=\left(X^{*}, \mathrm{w}^{*}\right)^{*}$. Since each $\mathrm{w}^{*}$ continuous linear functional $\phi$ is defined, for some $x \in X$, by the equation $\phi(f)=f(x)$, all $f \in X^{*}$, we can, by using the suggestive notation $\phi \in X$, state the following:

Theorem 1. An l.t.s. $X$ is pseudo-complete if and only if $\left(X^{*}, e-w^{*}\right)^{*} \subset X$.

So far, at least, little connection has been supplied between completeness and pseudo-completeness. Indeed, small motivation has been provided for a study of pseudo-completeness. The author first became really interested in it in connection with work on weak compactness and here the concept is useful, as shall be seen in our Corollary 3.3. Aside from this, the rather curious property (see [1, Theorem 1.4], [19, Theorem 5], and [15, Theorem 8.10]) that a linear subspace $L$ of the adjoint of a Banach space $X$ is $\mathrm{w}^{*}$ closed if and only if $L \cap S$ is $\mathrm{w}^{*}$ closed, where $S$ is the closed unit sphere of $X^{*}$, provides one with the natural question: for what kind of 1.t.s. $X$ can a generalization of this theorem be stated and proved? A study of the e-w* topol- 
ogy (here normed sphere is replaced by equicontinuous subset) provides background for a generalization, and a more detailed investigation is made of this question in Part 3. We give a partial answer in the next theorem as well as a characterization of pseudo-completeness which connects it with completeness. For this theorem (and for later work) we need the following lemma. Recall that if $(X, \mathcal{\tau})$ is an l.t.s., $\mathcal{T}_{c}$ is used to denote the topology for $X$ whose local base consists of all convex $\mathcal{T}$ neighborhoods of 0 . It is easily seen that $\mathcal{T}_{c}$ is coarser than $\mathcal{T}$ and that a set $S$ of linear functionals on $X$ is an equicontinuous family with respect to $\mathcal{T}$ if and only if the convex set $\{x:|f(x)| \leqq 1$ for all $f$ in $S\}$ is a $\mathcal{T}_{c}$ neighborhood of 0 . These remarks suffice to prove

Lemma 3. If $(X, \mathcal{T})$ is an l.t.s., then $(X, \mathcal{T})^{*}=\left(X, \mathcal{T}_{c}\right)^{*}$ and the subsets of $(X, \mathcal{T})^{*}$ which are equicontinuous with respect to $\mathcal{T}$ and $\mathcal{T}_{c}$ coincide.

TheOREM 2. If $(X, \mathcal{T})$ is an l.t.s., then $(X, \mathcal{T})$ is pseudo-complete if and only if $\left(X, \mathcal{T}_{c}\right)$ is complete.

Proof. Suppose first that $(X, \mathcal{T})$ is pseudo-complete and let $\left\{x_{a}: a \in A\right\}$ be a $\mathcal{T}_{c}$ cauchy net. Note that $X^{*}$ and $S(X)$ may be referred to without ambiguity by Lemma 3 . Then $\left\{x_{a}: a \in A\right\}$ converges pointwise on $X^{*}$ to a linear functional $\phi$ and converges uniformly to $\phi$ on each $S \in S(X)$. It is then clear by Lemma 1 that $\phi \in\left(X^{*}, \mathrm{e}-\mathrm{w}^{*}\right)^{*}$ and thus there is by Theorem 1 an $x \in X$ such that $\phi(f)=f(x)$, for all $f \in X^{*}$. To show $\left\{x_{a}: a \in A\right\}$ converges to $x$ with respect to $\mathcal{T}_{c}$ let $V$ be a $\mathcal{T}_{c}$ neighborhood of 0 . Since $\mathcal{T}_{c}$ is locally convex and $\left(X, \mathcal{T}_{c}\right)^{*}=(X, \mathcal{T})^{*}$, we may assume there exists a $\mathcal{T}$ neighborhood $U$ of 0 such that $\left(U^{0}\right)_{0}$ is contained in $V$. Since $\left\{x_{a}: a \in A\right\}$ converges uniformly to $\phi$ on each element of $S(X)$ there is $b \in A$ such that $a \geqq b$ implies $\left|f\left(x_{a}\right)-\phi(f)\right| \leqq 1$, for all $f \in U^{0}$; thus $\left|f\left(x_{a}-x\right)\right| \leqq 1$, all $f \in U^{0}$. Therefore $\left(x_{a}-x\right) \in\left(U^{0}\right)_{0} \subset V$, all $a \geqq b$, and $\left\{x_{a}: a \in A\right\}$ converges to $x$ with respect to $\mathcal{T}_{c}$.

Conversely, suppose $\left(X, \mathcal{T}_{c}\right)$ is complete and let $\phi$ be any e-w* continuous linear functional on $X^{*}$. By Lemma $2, \phi$ is $\mathrm{w}^{*}$ continuous on each element of $S(X)$; it then follows from a result of Grothendieck [12, p. 606] that (since $\left(X, \mathcal{V}_{c}\right)$ is complete) there is some element $x$ of $X$ such that $\phi(f)=f(x)$ holds for all $f \in\left(X, \mathcal{T}_{c}\right)^{*}=X^{*}$. But then $\phi$ is $\mathrm{w}^{*}$ continuous and we have shown that $\left(X^{*}, \mathrm{e}-\mathrm{w}^{*}\right)^{*} \subset X$; hence, by Theorem $1, X$ is pseudo-complete. This completes the proof.

4. Weak compactness. Several definitions needed for this section are found in the following paragraph.

If $(X, \mathcal{T})$ is an 1.t.s., recall that $X^{*}$ (more precisely $\left.(X, \mathcal{T})^{*}\right)$ denotes the adjoint space of $(X, \mathcal{T})$ consisting of all $\mathcal{\tau}$ continuous linear functionals on $X$. The cartesian product of $X^{*}$ copies of the real numbers, with the product topology, is always denoted by $R^{X^{*}}$, and the Mackey topology for $X$ is the 
finest locally convex topology for $X$ having $(X, \mathcal{T})^{*}$ as adjoint (alternatively, this topology is described as the topology for $X$ of uniform convergence on $\mathrm{w}^{*}$ compact, convex, and symmetric subsets of $\left.(X, \mathcal{\tau})^{*}\right)$. If $\phi$ is a real valued function on $X^{*}$, the null space of $\phi$ is denoted by $K_{\phi}$. Finally, the convex [closed convex] hull of a subset $A$ of $X$ is the smallest convex [closed convex] set containing $A$, and $A-\mathcal{T}$ denotes the $\mathcal{T}$ closure of $A$.

Lemma 4. Let $X$ be an l.t.s., $M \subset X, S \subset X^{*}$, and suppose $\phi$ is a point in the $R^{X^{*}}$ closure of $M$ and $f_{0}$ is a point in $\left(K_{\phi} \cap S\right) \rightarrow^{*}$. Then there exist sequences $\left\{x_{n}: n=0,1,2, \cdots\right\}$ in $M$ and $\left\{f_{m}: m=1,2, \cdots\right\}$ in $K_{\phi} \cap S$ such that (a) $\left|\phi\left(f_{0}\right)-f_{0}\left(x_{n}\right)\right|<1 / 2^{n}, n=0,1,2, \ldots$; (b) $\left|f_{m}\left(x_{n}\right)-f_{0}\left(x_{n}\right)\right|<1 / 2^{m-1}$, $0 \leqq n \leqq m-1$ and each $m \geqq 1$; and (c) $\left|f_{m}\left(x_{n}\right)\right|<1 / 2^{n}, 1 \leqq m \leqq n$ and each $n \geqq 1$.

Proof. The proof is easily made by alternate appeal to properties (A) and $(B)$ below and will be omitted:

(A) Since $\phi$ is in the $R^{X^{*}}$ closure of $M$, it is a linear functional on $X^{*}$ and we know that $\epsilon>0, f_{1}, \cdots, f_{n} \in X^{*}$ implies there exists $x \in M$ such that $\left|\phi\left(f_{i}\right)-f_{i}(x)\right|<\epsilon, i=1,2, \cdots, n$.

(B) Since $f_{0} \in\left(K_{\phi} \cap S\right)^{-w^{*}}$ we have that $\epsilon>0, x_{1}, \cdots, x_{n} \in X$ implies there exists $f \in\left(K_{\phi} \cap S\right)$ such that $\left|f\left(x_{i}\right)-f_{0}\left(x_{i}\right)\right|<\epsilon, i=1,2, \cdots, n$.

THEOREM 3. Let $(X, \mathcal{T})$ be an l.t.s. and suppose $M$ is a mean compact subset (see \$2) of $X$. Then if $\phi$ is in the $R^{X^{*}}$ closure of $M$ and $S \subset X^{*}$ is equicontinuous on the weakly closed convex hull of $M$ with respect to its relative $\mathcal{T}_{c}$ topology, we have $\left(K_{\phi} \cap S\right)^{-w^{*}} \subset K_{\phi}$.

Proof. Let $\left\{x_{n}\right\}$ and $\left\{f_{m}\right\}$ be the sequences in $M$ and $\left(K_{\phi} \cap S\right)$ respectively provided by Lemma 4 . Since $M$ is mean compact there exists $z_{0} \in X$ such that $\lim _{n}$ inf $f\left(x_{n}\right) \leqq f\left(z_{0}\right) \leqq \lim _{n}$ sup $f\left(x_{n}\right)$, all $f$ in $X^{*}=\left(X^{*}, \mathcal{T}\right)^{*}$. Let $C$ be the weakly closed convex hull of the set $\left\{x_{n}: n=0,1,2, \ldots\right\}$. Since $M$ is mean compact, it is clear by the separation theorem [17, p. 466], applied to the weak topology in $X$, that $z_{0} \in C$. It then follows from (a) of Lemma 4 that $\left\{f_{0}\left(x_{n}\right)\right\}$ converges to $\phi\left(f_{0}\right)$ and, since $\lim _{n} \inf f_{0}\left(x_{n}\right) \leqq f_{0}\left(z_{0}\right) \leqq \lim _{n} \sup f_{0}\left(x_{n}\right)$, that

$$
f_{0}\left(z_{0}\right)=\phi\left(f_{0}\right) .
$$

From (c) of Lemma 4 we see that for each $m,\left\{f_{m}\left(x_{n}\right)\right\}$ converges to 0 with $n$, and since $\lim _{n} \inf f_{m}\left(x_{n}\right) \leqq f_{m}\left(z_{0}\right) \leqq \lim _{n} \sup f_{m}\left(x_{n}\right)$, it follows that

$$
f_{m}\left(z_{0}\right)=0 \text {, for all positive integers } m \text {. }
$$

Finally, from (b), $\left\{f_{m}\left(x_{n}\right)\right\}$ converges to $f_{0}\left(x_{n}\right)$ for each $n=0,1,2, \cdots$.

Since $\mathcal{T}_{c}$ is locally convex, the convex hull $H$ of the set $\left\{x_{n}: n=0,1,2, \cdots\right\}$ has its $\mathcal{T}_{c}$ closure coinciding with its closure in the weak topology generated by $\mathcal{T}_{c}$. But since $\left(X, \mathcal{T}_{c}\right)^{*}=(X, \mathcal{T})^{*}$, it follows then that the $\mathcal{T}_{c}$ closure of $H$ is $C$. Since the sequence $\left\{f_{m}\right\}$ of linear functionals converges to $f_{0}$ at each 
$x_{n}$, it converges to $f_{0}$ at each point of $H$. Since $H$ is $\mathcal{G}_{c}$ dense in $C$ and the sequence $\left\{f_{m}\right\}$ is also equicontinuous on $C$ with respect to the $\mathcal{T}_{c}$ relative topology, it follows from a standard convergence theorem that $\left\{f_{m}\right\}$ converges to $f_{0}$ at every point of $C$, and in particular at $z_{0}$. But then $\left(^{* *}\right)$ shows $f_{0}\left(z_{0}\right)=0$, and $\left(^{*}\right)$ implies $\phi\left(f_{0}\right)=0$. This completes the proof of the theorem.

REMARK. The equicontinuity hypothesis in the above theorem is fulfilled if $S$ is equicontinuous on $X$, since $S$ is then equicontinuous on $\left(X, \tau_{c}\right)$ by Lemma 3.

Corollary 3.1. Let $X$ be an l.t.s., $M$ be a mean compact subset of $X$, and let $A$ denote the Mackey closed convex hull of $M$. If $\phi$ is in the $R^{X^{*}}$ closure of $M$ and $S \subset X^{*}$ is equicontinuous on $A$ with respect to its relative Mackey topology, then $\left(K_{\phi} \cap S\right)^{-w^{*}} \subset K_{\phi}$. If $S$ is also $w^{*}$ closed, then $K_{\phi} \cap S$ is $w^{*}$ closed.

Proof. The proof is clear by the theorem when one recalls that the Mackey topology is a locally convex topology having $X^{*}$ as adjoint [2].

Corollary 3.2. Let $X$ be an l.t.s., $M \subset X$ be mean compact, and denote by $B$ the $R^{X^{*}}$ closure of $M$. Then $B C\left(X^{*}, \mathcal{T}\right)^{*}$, where $\mathcal{T}$ is the finest topology for $X^{*}$ which coincides with $w^{*}$ on $w^{*}$ compact, convex, and symmetric subsets; hence if $X$ is pseudo-complete with respect to its Mackey topology we have $B \subset X$.

Proof. Fix $\phi \in B$. By definition of the Mackey topology we have this characterization: a subset of $X^{*}$ is equicontinuous with respect to the Mackey topology of $X$ if and only if it is a subset of some $w^{*}$ compact, convex, and symmetric set; hence $S(Y)$, where $Y=(X$, Mackey), is precisely the family of all $\mathrm{w}^{*}$ compact, convex, and symmetric subsets of $X^{*}$. From Corollary 3.1 it is clear that $K_{\phi} \cap S$ is w* closed for every $S \in S(Y)$; i.e., $K_{\phi}$ is closed in the e-w* topology in $X^{*}$ generated by the Mackey topology of $X$, that is, in the finest topology $\mathcal{T}$ for $X^{*}$ that coincides with w* on each $S \in S(Y)$. From Lemma 2 it is clear that $\phi \in\left(X^{*}, \mathcal{T}\right)^{*}$. If $X$ is pseudo-complete in its.Mackey topology, then $\left(X^{*}, \tau\right){ }^{*} \subset X$ by Theorem 1 , and the proof is completed.

The previous corollaries have led up to the next (and main) result of this section, our theorem on weak compactness. Here, in Corollary 3.3, we use the concept pseudo-completeness to connect eight types of compactness.

REMARK. If $\mathcal{T}$ and $\mathcal{T}^{\prime}$ are two linear topologies for $X$ such that $(X, \mathcal{T})^{*}$ $=\left(X, \mathcal{T}^{\prime}\right)^{*}$ and $\mathcal{T}^{\prime}$ is finer than $\mathcal{T}_{c}$ (note that $\mathcal{T}^{\prime}$ finer than $\mathcal{T}$ implies this last condition), then $(X, \mathcal{T})$ pseudo-complete implies $\left(X, \mathcal{O}^{\prime}\right)$ is pseudo-complete. In fact, if $\phi$ is a linear functional on $X^{*}$ which is $\mathrm{w}^{*}$ continuous on each $\mathrm{w}^{*}$ closed, convex, and symmetric set which is equicontinuous with respect to $\mathcal{T}^{\prime}$, let $S$ be w* closed, convex, symmetric and equicontinuous with respect to $\mathcal{T}$. It is then, by Lemma 3 , also equicontinuous with respect to $\mathcal{T}_{c}$; hence also equicontinuous with respect to $\mathcal{T}^{\prime}$, since $\mathcal{T}_{c}$ is coarser than $\mathcal{T}^{\prime}$. But then, by hypothesis, the restriction of $\phi$ to $S$ is w* continuous; hence $\phi \in X$ by Lemma 
2 and Theorem 1 , since $(X, \mathcal{T})$ is pseudo-complete. But then Lemma 2 and Theorem 1 imply $\left(X, \mathcal{T}^{\prime}\right)$ is pseudo-complete.

In particular, this remark is applicable to the case where $\mathcal{T}^{\prime}$ is the Mackey topology of $X$; it is thus clear that $X$ is pseudo-complete with respect to its Mackey topology (or, equivalently by Theorem 2, complete) whenever $X$ is pseudo-complete in its given topology.

Corollary 3.3. Let $X$ be an l.t.s., $M$ a subset of $X$, and consider these conditions on $M$ (see §2): (1) the weak closure of $M$ is weakly compact; (2) the weak closure of $M$ is weakly Fréchet compact; (3) the weak closure of $M$ is weakly cluster point compact; (4) $M$ is weakly Fréchet compact in $X$; (5) $M$ is weakly cluster point compact in $X$; (6) $M$ is weakly semi-cluster point compact in $X$; (7) given any sequence $\left\{x_{n}\right\}$ of points of $M$ there exists a point $x \in X$ such that $f \in X^{*}$ implies there is a subsequence $\left\{x_{n_{i}}\right\}$ of $\left\{x_{n}\right\}$ such that $\left\{f\left(x_{n_{i}}\right)\right\}$ converges to $f(x)$; (8) $M$ is mean compact; and (9) $M$ is weakly sequentially compact in $X$.

Then: (a) when $X$ is complete or pseudo-complete with respect to its Mackey topology, (1) through (8) are equivalent; (b) there exists a complete locally convex Hausdorff l.t.s. whose topology is the Mackey topology (hence (1) through (8) are equivalent by (a)) but none of (1) through (8) implies (9); and (c) X an $F$ or $L F$ space implies (1) through (9) are equivalent.

Proof. (a) Let $M \subset X$ be mean compact. We shall prove that the weak closure of $M$ is weakly compact, hence (see §1) (1) through (8) are equivalent. The mean compactness of $M$ implies that $f(M)$ is bounded for each $f \in X^{*}$ and $f(M) \subset I_{f}$, where $I_{f}$ is a closed and bounded interval in the reals. But then the $R^{X^{*}}$ closure of $M$ is a subset of the compact set $P_{f \in X^{*} I_{f}} \subset R^{X^{*}}$. The preceding Corollary 3.2 coupled with Theorem 2 shows that the $R^{X^{*}}$ closure of $M$ is contained in $X$, hence the weak closure of $M$ is the $R^{X^{*}}$ closure; the latter being compact in $R^{X^{*}}$, it is thus clear that the weak closure of $M$ is weakly compact.

(b) The example we give was cited by Grothendieck for the same purpose [11]. Let $X$ be the product of the reals over the closed unit interval of real numbers, with the product topology. $X$ is a complete locally convex Hausdorff space whose topology is the Mackey topology. Hence, by part (a) of this theorem, (1) through (8) are equivalent. However, Tychonoff has shown $[28$, p. 764$]$ that there exists in $X$ a subset whose closure is compact but which is not sequentially compact in $X$. The fact that the topology of $X$ is the weak topology [14] then concludes part (b).

(c) It was proved in [6, Theorem 3] that the topology of any $F$ or $L F$ space is the Mackey topology and that these spaces are complete, thus (1) through (8) are equivalent by (a). The Dieudonné and Schwartz result [6, pp. 88-89] shows that (1), (4), and (9) are equivalent in such spaces; hence all nine conditions coincide. This completes the proof.

In concluding this section it might be worthwhile to point out that the 
space $X$ of the preceding part (b) can be used to construct an example showing that weak sequential compactness does not always imply weak compactness (this does not contradict the results of the above corollary, for there we are concerned with weak sequential compactness in $X$ and with the weak compactness of the weak closure of $M$ ). In fact, $X$ contains a subset which is weakly sequentially compact but is not weakly closed.

\section{Part 2. Certain topologies in linear topological spaces}

5. A general method of definition of topologies. Let $\mathcal{S}$ and $\mathcal{T}$ be collections of subsets of an arbitrary set $X$. Then $(S-\mathcal{T})$ is, by definition, the collection of all subsets $E$ of $X$ for which $S \in \mathcal{S}$ implies there exists $T \in \mathcal{T}$ such that $E \cap S=T \cap S$.

Remark. For any family $\mathcal{A}$ of subsets of $X$ let $\mathcal{A}$-denote the collection of all complements of members of $\mathcal{A}$. Then $(\mathcal{S}-\mathcal{T})^{\wedge}=\left(\mathcal{S}-\mathcal{T}^{\wedge}\right)$.

TheOREM 4. (1) If $\mathcal{T}$ is a topology for $X$, so also is $(\mathcal{S}-\mathcal{T})$ and the latter is, in fact, the finest topology coarser than $\mathcal{T}$ on each member of $\mathcal{S}$; since $\mathcal{T} \subset(\mathcal{S}-\mathcal{T})$, clearly $(\mathcal{S}-\mathcal{T})$ is the finest topology that coincides with $\mathcal{T}$ on each element of $\mathcal{S}$.

(2) If $\mathcal{S}^{\prime}$ and $S$ are two collections of subsets of $X$ such that each $S^{\prime} \in \mathcal{S}^{\prime}$ is contained in some $S \in \mathcal{S}$, then $(\mathcal{S}-\mathcal{T}) \subset\left(\mathcal{S}^{\prime}-\mathcal{T}\right)$; in particular, if $\mathcal{S}^{\prime}$ is the collection of all sets each of which is a subset of some $S \in \mathcal{S}$, then $(\mathcal{S}-\mathcal{T})$ $=\left(\boldsymbol{S}^{\prime}-\mathcal{T}\right)$.

(3) If $\mathcal{S}, \mathcal{T}, \mathcal{T}^{\prime}$ are collections of subsets such that $\mathcal{} \subset \mathcal{T}^{\prime}$, then $(\mathcal{S}-\mathcal{T})$ $C\left(S-\mathcal{T}^{\prime}\right)$.

Corollary 4.1. If $Y$ is an l.t.s. and $E \subset Y^{*}$, then $E$ is e-w* open [e-w* closed] if and only if $E \cap U^{0}$ is open [closed] in the relative w* topology of $U^{0}$ for every neighborhood $U$ of 0 in $X$.

Proof. In Theorem 4, (2), if one takes $S$ to be the collection of all polars in $Y^{*}=X$ of neighborhoods of 0 in $Y$, and $S^{\prime}$ to be all equicontinuous subsets of $Y^{*}$, then the statement regarding openness is clear; the remainder of the corollary then follows from this and the remark preceding the theorem.

The preceding theorem exhibits $(S-\mathcal{T})$ as the finest topology coinciding with $\mathcal{T}$ on each element of $\mathcal{S}$. When the space $X$ is a linear space and $\mathcal{T}$ is a semi-linear topology (i.e., for $x, y \in X$ and $a$ real, the functions $x+y$ and $a x$ are each $\mathcal{T}$ continuous in each variable separately), certain additional information concerning $(\mathcal{S}-\mathcal{T})$ is easily established.

THEOREM 5. Let $X$ be a linear space and $\mathcal{T}$ be a semi-linear topology for $X$. Suppose $S$ is a family of subsets of $X$ such that (i) each translate of a member of $S$ is contained in a member of $S$; (ii) $S$ is closed under nonzero scalar multiplication; and (iii) given any nonzero $x \in X$ there is an $\epsilon>0$ and some $S \in \mathcal{S}$ such that ax $\in S$, for all a such that $|a|<\epsilon$. Under these conditions $(\mathcal{S}-\mathcal{T})$ is a semilinear topology. 
Proof. The proofs that $x+y$ is $(\mathcal{S}-\mathcal{T})$ continuous in each variable separately and that $a x$ is $(\mathcal{S}-\mathcal{T})$ continuous in $x$ for each $a$ are direct. Similarly, it is easily shown that $a x$ is $(S-\mathcal{T})$ continuous in $a$ at $x=0$; therefore let $x_{0} \in X$, with $x_{0} \neq 0$. Since $x+y$ is $(S-\tau)$ continuous in each variable separately, it clearly suffices to show $a x_{0}$ is continuous at $a=0$. By hypothesis, there exists $\epsilon>0$ and some $S \in \mathcal{S}$ such that if $A=\left\{a x_{0}:|a|<\epsilon\right\}$, then $A \subset S$. Now let $E$ be any $(S-\mathcal{T})$ neighborhood of 0 . There is then some $T$ in $\mathcal{T}$ such that $E \cap S=T \cap S$; hence $E \cap A \subset E \cap S=T \cap S$, and so $E \cap A=E \cap A$ $\cap T \cap S=E \cap S \cap T \cap A=T \cap S \cap T \cap A=T \cap S \cap A=T \cap A$, the latter since $A \subset S$. But $0 \in E$ and $0 x_{0}=0 \in S$ and thus $0 \in T$. Since $a x_{0}$ is $\mathcal{T}$ continuous at $a=0$, there exists $\delta>0$ such that $|a|<\delta$ implies $a x_{0} \in T$. Therefore

$$
|a|<\min (\delta, \epsilon)
$$

implies $a x_{0} \in T \cap A$; thus $a x_{0} \in E \cap A \subset E$ and $a x_{0}$ is $(\mathcal{S}-\mathcal{T})$ continuous at $a=0$. This completes the proof.

If $X$ is a linear space and $\mathcal{T}$ a family of sets in $X$ we denote by $\mathcal{T}^{c}$ the family of all subsets $E$ of $X$ such that $E$ is the union of a collection of convex elements of $\mathcal{T}$. When $\mathcal{T}$ is a topology the sets $(X, \mathcal{T})^{*}$ and $\left(X, \mathcal{T}^{c}\right)^{*}$ are, as in $\S 3$, respectively the spaces of $\mathcal{T}$ and $\mathcal{\sigma}^{c}$ continuous linear functionals. The following theorem is easily proved and its proof is omitted.

THEOREM 6. If $\mathcal{T}$ is a topology, then $\mathcal{T}^{c}$ is a locally convex topology and is the finest such topology which is coarser than $\mathcal{T}$. In addition, the spaces $(X, \mathcal{\tau})^{*}$ and $\left(X, \mathcal{T}^{c}\right)^{*}$ are identical.

TheOREм 7. If $\mathcal{T}$ is a semi-linear topology then $\mathcal{T}^{\circ}$ is a linear topology and therefore $\left(X, \mathcal{T}^{c}\right)$ is a locally convex l.t.s. having for a local base all convex and symmetric $\mathcal{T}$ neighborhoods of 0 ; in this case, $\mathcal{T}^{c}$ coincides with $\mathcal{T}_{c}$.

Proof. The proof that $\tau^{c}$ is semi-linear is a direct and obvious one. From the definition of $\mathcal{T}^{c}$ it is clear that the family of all convex $\mathcal{T}$ neighborhoods of 0 form a local base for $\mathcal{T}^{c}$. It then follows from a well known result (e.g., see $\left[4\right.$, p. 57]) that $\left(X, \mathcal{\sigma}^{c}\right)$ is a locally convex l.t.s. But then, since $\mathcal{\sigma}^{c}$ and $\tau_{c}$ have the same local base, these two topologies coincide. Finally, since $\mathcal{T}^{c}$ is a linear topology, it is clear that the local base for $\mathcal{T}^{c}$ can be assumed to consist of all convex and symmetric $\mathcal{T}$ neighborhoods of 0 .

THEOREM 8. Let $X$ be a linear space with $S$ and $\tau$ collections of subsets of $X$. Then: (1) if $\mathcal{T}$ is a locally convex topology, $(\mathcal{S}-\mathcal{T})^{c}$ is the finest locally convex topology which coincides with $\mathcal{T}$ on each element of $\mathcal{S}$; (2) if $\mathcal{T}$ and $\mathcal{S}$ satisfy the hypotheses of Theorem $5,(\mathcal{S}-\mathcal{T})_{c}\left[=(S-\mathcal{T})^{c}\right.$, by Theorem 7] is a locally convex and linear topology having a local base consisting of all convex symmetric sets $W$ such that $S \in \mathcal{S}, 0 \in S$ implies $W \supset S \cap V$, for some $\mathcal{T}$ neighborhood $V$ of 0 ; and (3) if $\mathcal{T}$ and $\mathcal{S}$ satisfy the conditions which the hypotheses of Theorem 5 impose, if $\mathcal{S}^{\prime} C S$ is a collection of subsets satisfying the conditions on $S$, and if each 
$S \in \mathcal{S}$ which contains 0 is contained in some $S^{\prime} \in \mathcal{S}^{\prime}$, we have $(\mathcal{S}-\mathcal{T})_{c}=\left(\mathcal{S}^{\prime}-\mathcal{T}\right)_{c}$.

Proof. The proof of (1) follows easily from Theorems 4 and 6. To establish (2) we show first that the family $\mathscr{W}$ of sets consisting of all convex symmetric sets $W$ such that $S \in \mathcal{S}, 0 \in S$ implies there exists a $\mathcal{T}$ neighborhood $V$ of 0 for which $W \supset S \cap V$ is a local base for a locally convex linear topology; and, secondly, that the topology $T(\mathscr{W})$ having $\mathscr{W}$ as a local base is coarser than $(\mathcal{S}-\mathcal{T})_{c}$ (note that $(\mathcal{S}-\mathcal{T})_{c}$ is obviously coarser than $T(\mathscr{W})$ by Theorem 7 since each member of the local base for $(\mathcal{S}-\mathcal{T})_{c}$ is an element of $\mathscr{W}$ ). Now it is easily verified that $W$ is closed under finite intersections and, if one makes use of (ii) of Theorem 5 , that $\mathscr{W}$ is also closed under nonzero scalar multiplication. Since each element $W$ of $W$ is convex and symmetric, it is sufficient [see 4, p. 57] to prove each $W$ satisfies this condition: given any nonzero $x \in X$, there is an $\epsilon>0$ such that $a x \in W$ for all scalars $a$ for which $|a|<\epsilon$. By Theorem 5, (iii) there is an $\epsilon^{\prime}>0$ and some $S \in \mathcal{S}$ such that $a x \in S$ whenever $|a|<\epsilon^{\prime}$; since $0 \in S$ and $W \in \mathscr{W}, W \supset V \cap S$ for some $\mathcal{T}$ neighborhood $V$ of 0 . But $\mathcal{T}$ is semi-linear and therefore there is a $\delta>0$ for which $|a|<\delta$ implies $a x \in V$. Let $\epsilon=\min \left(\epsilon^{\prime}, \delta\right)$; then $|a|<\epsilon$ implies $a x \in V \cap S \subset W$.

Secondly, we prove $T(\mathscr{W})$ is coarser than $(\mathcal{S}-\mathcal{G})_{c}$. Since $T(\mathscr{W})$ is locally convex it is sufficient, by Theorem 6 (applied to $(S-\mathcal{T})$ ) and by Theorem 4 , (1), to prove $T(\mathscr{W})$ is coarser on each $S \in \mathcal{S}$ than is $\mathcal{T}$. Finally, it suffices by definition of $\mathscr{W}$ and by (i) of Theorem 5 to show that if $S \in \mathcal{S}$ and $0 \in S$ and if $\left\{x_{a}: a \in A\right\}$ is a net in $S$ converging to 0 with respect to the $\mathcal{T}$ relative topology of $S$, then $\left\{x_{a}: a \in A\right\}$ also converges to 0 with respect to the $T(\mathscr{W})$ relative topology of $S$. For this purpose, fix $W \in W$; then $W \supset V \cap S$ for some $\mathcal{T}$ neighborhood $V$ of 0 , hence there exists $b \in A$ such that $a \geqq b$ implies $x_{a} \in V \cap S \subset W$. This shows that $\left\{x_{a}: a \in A\right\}$ converges to 0 for $T(\mathscr{W})$ and completes the proof of (2).

To prove (3) note that, since $S^{\prime} C S$, it is clear by Theorem $4,(2)$, that $(S-\mathcal{T}) \subset\left(S^{\prime}-\mathcal{T}\right)$; hence $(S-\mathcal{T})_{c} \subset\left(S^{\prime}-\mathcal{T}\right)_{c}$. For the reverse inclusion, let $W$ be a member of the local base for $\left(\boldsymbol{S}^{\prime}-\mathcal{T}\right)_{c}$, where $W$ can be assumed to be convex and symmetric. Then, if $S \in \mathcal{S}$ and $0 \in \mathcal{S}$, there exists by hypothesis an $S^{\prime} \in \mathcal{S}^{\prime}$ for which $S \subset S^{\prime}$; hence $W \supset W \cap S=W \cap S \cap S^{\prime}=W \cap S^{\prime} \cap S \supset V$ $\cap S^{\prime} \cap S$, where $V$ is a $\mathcal{T}$ neighborhood of 0 . But since $S \subset S^{\prime}$, we have $V \cap S^{\prime} \cap S=V \cap S$, and thus $W$ is a member of the local base for $(\mathcal{S}-\mathcal{T})_{c}$. It is then clear that $\left(S^{\prime}-\mathcal{T}\right)_{c} \subset(S-\mathcal{T})_{c}$.

COROLlary 8.1. If $Y$ is an l.t.s. and if $S$ and $\mathcal{S}^{\prime}$ denote respectively the families of all equicontinuous subsets of $Y^{*}$ and of all polars in $Y^{*}$ of neighborhoods of 0 in $Y$, then $\left(S-w^{*}\right)_{c}=\left(S^{\prime}-w^{*}\right)_{c}$.

Proof. If we let the $X$ of Theorem 8, (3) be $Y^{*}$ and $\mathcal{T}=w^{*}$ it is easy to see that $S, S^{\prime}$, and $\mathcal{T}$ satisfy the hypotheses of that theorem.

It should be noted that when $\delta$ and $\mathcal{T}$ are collections of subsets of a linear space $X$ satisfying the hypotheses of Theorem 5 it is false, in general, that 
$\left(S-\mathcal{T}_{c}\right)=(S-\mathcal{T})_{c}$, even when $\mathcal{T}$ is a locally convex linear topology for $X$ (and therefore $\mathcal{T}_{c}=\mathcal{T}$ ). In fact, $\left(\mathcal{S}-\mathcal{T}_{c}\right.$ ) is not necessarily a linear topology. To see this let $S$ be the family of all one-dimensional linear manifolds (i.e., translates of one-dimensional linear subspaces) of a linear space $X$ and $\mathcal{T}$ be a locally convex linear topology for $X$ which is Hausdorff. Since the $\mathcal{T}$ relative topology of each finite-dimensional linear subspace is that of the reals when $\mathcal{T}$ is Hausdorff and linear [29], it is the same for each $S \in \mathcal{S}$. But then the topology $(S-\tau)$ is simply the radial topology $(H \subset X$ is a radial neighborhood of $y \in X$ if and only if $x \neq y$ implies there exists an $\epsilon>0$ for which $[y+L(x)] \cap H \supset\{y+a x:|a|<\epsilon\}$, where $L(x)$ is the linear subspace spanned by the single point $x$ ). However, Klee has shown [17] that the radial topology is not always linear.

Another example we give here exhibits $(\mathcal{S}-\mathcal{T})$ as a linear topology which is not locally convex; hence $(S-\mathcal{T})$ and $(S-\mathcal{T})_{c}$ are distinct. More specifically, let $(X, \mathcal{T})$ be any pseudo-metrisable l.t.s. which is not locally convex, and let $\delta$ be the family of $\mathcal{T}$ compact subsets of $X$. Each of $\mathcal{S}$ and $\mathcal{\tau}$ satisfy the hypotheses of Theorem 5 ; hence $(S-\mathcal{T})$ is a semi-linear topology, and $(\mathcal{S}-\mathcal{T})_{c}$ is a locally convex linear topology by Theorem 8 , (2). In fact, it is well known that here $(\mathcal{S}-\mathcal{T})=\mathcal{T}$ (this is true when $(X, \mathcal{T})$ is any topological space having a countable base at each point $[10])$. But then $(\mathcal{S}-\mathcal{T})$ is a nonlocally convex and linear topology while $(\delta-\mathcal{T})_{c}$ is locally convex and linear.

6. Uniform convergence topologies. Let $(X, \mathcal{\tau})$ be an 1.t.s., $U$ a local base for $\mathcal{T}$, and $G$ be an admissible family [2] of sets in $X$ (i.e., $G$ is closed under finite unions, under nonzero scalar multiplication, and each element of $G$ is weakly bounded). When $G$ is such a family, the topology u.c. $G$ is defined as the locally convex and linear topology for $(X, \mathcal{T})^{*}$ having a local base consisting of all polars (in $(X, \mathcal{T})^{*}$ ) of elements of $G$ (see $[30 ; 6]$ ). A set $B$ in $X$ is $G-\tau$ totally bounded if $U \in V$ implies there exists $G \in G$ such that $B \subset G+U$.

The proofs of the next three lemmas are all direct and are omitted. Recall that for $E \subset X$ and $F \subset X^{*}, E^{0}$ and $F_{0}$ denote respectively the polars of $E$ in $X^{*}$ and $F$ in $X$.

Lemma 5. (1) If $G$ consists of all finite sets of $X$, then the $G-\tau$ totally bounded sets are simply the $\mathcal{T}$ totally bounded sets.

(2) The family $\mathcal{B}$ of all $G-\mathcal{T}$ totally bounded sets in $X$ is admissible, and $G \subset B$; in particular, when $\mathcal{G}$ is a locally convex topology for $X$ and $G$ consists of all sets bounded with respect to $\mathcal{T}$, then the $G-\mathcal{T}$ totally bounded sets coincide with the $\mathcal{T}$ bounded sets.

Lemma 6. If $A, B \subset X$, then $2(A+B)^{0} \supset(A \cup B)^{0}$; hence $\left[(A+B)^{0}\right]_{0}$ $C 2\left[(A \cup B)^{0}\right]_{0}$.

Lemma 7. If $A, B \subset X$, then $\left[(A \cup B)^{0}\right]_{0} \subset\left[\left(A^{0}\right)_{0}+\left(B^{0}\right)_{0}\right]^{-w}$. 
Lemma 8. If $G$ is an admissible family in $(X, \mathcal{T})$ and $\mathbb{B}$ is the family of all $G-\mathcal{T}$ totally bounded sets in $X$, then the topologies u.c. $\mathbb{B}$ and u.c. $G$ coincide on each equicontinuous set of $X^{*}$; hence also on each set of the form $U^{0}$, where $U$ is a member of the local base $U$ for $\mathcal{T}$.

Proof. Since $G \subset B$ by Lemma 5 , it is clear that u.c. $G$ is coarser than u.c. $\mathscr{B}$; in particular, it is coarser on each equicontinuous set. To prove that u.c. $\mathbb{B}$ is coarser than u.c. $G$ on any equicontinuous set $E$ it is sufficient, since equicontinuous sets, u.c. $G$ open sets, and u.c. $\mathcal{B}$ open sets are all invariant under translation, to consider only $E$ which contain 0 . Thus, if $N$ is a u.c. $B$ neighborhood of 0 , we wish to find a u.c. $G$ neighborhood $W$ of 0 for which $E \cap N \supset E \cap W$. But since $E$ is equicontinuous, we have $E \subset U^{0}$ for some $U \in U$. Since $E \cap N=U^{0} \cap E \cap N$ and $E \cap W=U^{0} \cap E \cap W$, the problem reduces to that of finding some $W$ such that $U^{0} \cap N \supset U^{\circ} \cap W$. Now $N$ is a neighborhood of 0 for u.c. $\mathcal{B}$ and therefore $N \supset B^{0}$ for some $B \in \mathcal{B}$; since $B \in \mathcal{B}$ it then follows from Lemma 6 that $U^{0} \cap B^{0} \supset U^{0} \cap G^{0}$ for some $G \in G$. We then set $W=G^{0}$, completing the first part of the lemma. Since each set $U^{0}(U \in V)$ is equicontinuous, it follows from the first part that u.c. $\mathbb{B}$ and u.c. $\mathcal{G}$ coincide on $U^{0}$.

TheOREM 9. In an l.t.s. $(X, \mathcal{T})$ with local base $V$ let $\mathcal{C}$ and $G$ be admissible families such that u.c. $\mathcal{C}$ is coarser on each $U^{0}(U \in \mathcal{U})$ than u.c. $G$. Let $\mathbb{B}$ be the family of all $G-\mathcal{T}$ totally bounded sets in $X$ and suppose $G$ has the following property: (*) $U \in \mathcal{U}$ implies there exists $V \in \mathcal{U}$ such that $G \in G$ implies there is some $G_{1} \in G$ for which $\left[\left(G^{0}\right)_{0}+\left(V^{0}\right)_{0}\right]^{-\omega} \subset G_{1}+U$. Then $\mathcal{C} \subset \mathbb{B}$ and hence u.c. $B$ is the finest u.c. topology in $X^{*}$ which coincides with u.c. $G$ on each equicontinuous subset of $X^{*}$.

Proof. We show first that, under these hypotheses, $\mathcal{C} \subset B$; i.e., each $C \in \mathcal{C}$ is $G-\mathcal{T}$ totally bounded. Let $U$ be any member of $U$ and let $V \in \mathcal{V}$ be as in $\left(^{*}\right)$. There is then, by hypothesis, a $G \in G$ such that $C^{0} \supset G^{0} \cap V^{0}$ $=(G \cup V)^{0}$; hence $C \subset\left(C^{0}\right)_{0} \subset\left[\left(G^{0}\right)_{0}+\left(V^{0}\right)_{0}\right]^{-w}$ by Lemma 7. But then $\left(^{*}\right)$ implies the latter is a subset of $G_{1}+U$, for some $G_{1} \in G$; hence $C$ is $G-\mathcal{T}$ totally bounded.

To complete the theorem, let $\mathfrak{H}$ be any admissible family in $X$ for which u.c. Te coincides with u.c. $G$ on each equicontinuous set. Since each $U^{0}$ $(U \in U)$ is equicontinuous and since u.c. $\mathfrak{H}$ coincides with u.c. $G$ on equicontinuous sets it is true, in particular, that u.c. $\mathcal{H C}$ is coarser on each $U^{0}$ than is u.c. $G$. It then follows by the first part that $\mathfrak{F C} \subset \mathbb{B}$ and therefore u.c. $\mathcal{F}$ is coarser than u.c. B. This completes the proof.

It perhaps should be remarked that our only application of the above theorem is to (1) and (2) of Theorem 10 which follows.

7. Further properties of $e-w^{*}$. The purpose which motivates the remainder of Part 2 is that of determining, and describing the relationship between, 
the following three (in general distinct) topologies for the adjoint of an 1.t.s. $X$ : the finest u.c. topology, the finest locally convex topology, and the finest topology, each being finest with respect to the property "each coincides with the $\mathrm{w}^{*}$ topology on equicontinuous subsets of $X^{*}$ " (or, what amounts to the same thing, each coincides with $\mathrm{w}^{*}$ on each element of $S(X)$; see Lemma 8 and Corollaries 8.1 and 4.1). The last topology mentioned above has already been determined as the topology e-w*; the theorems which follow give additional information concerning these three topologies. We state first a theorem (Lemma 9) whose proof is included for the sake of completeness.

Lemma 9. Let $Y$ be an l.t.s. and $\gamma$ be a locally convex linear topology for $Y$ such that $(Y, \gamma)^{*}=Y^{*}$, and suppose $W \subset Y$ is a radial neighborhood of 0 (see after Corollary 8.1) which is convex symmetric and such that $\left(W^{0}\right)_{0}$ is a $\gamma$ neighborhood of 0 . Then $W$ is a $\gamma$ neighborhood of 0 if and only if each linear functional on $Y$ which is bounded on $W$ is $\gamma$ continuous.

Proof. It is clearly sufficient to prove that $W$ is a $\gamma$ neighborhood of 0 when each linear functional on $Y$ which is bounded on $W$ is $\gamma$ continuous. Let $p$ be the pseudo-norm determined by $W$. By the Hahn-Banach Theorem, for each $x \in Y$ there is a linear functional $f_{x}$ on $Y$ such that $f_{x}(x)=p(x)$ and $\sup _{y \in Y}\left|f_{x}(y)\right| \leqq p(y)$. Since $W \subset\{x: p(x) \leqq 1\}$, for each $x \in Y$ it is true that $\sup _{y} \in W\left|f_{x}(y)\right| \leqq 1$; hence $f_{x}$ is bounded on $W$. But then, by hypothesis, $f_{x} \in Y^{*}$, and therefore $f_{x} \in W^{0}$. Now if $x \in\left(W^{0}\right)_{0}$, we have $p(x)=f_{x}(x) \leqq 1$, and thus $\{x: p(x) \leqq 1\}$ contains the $\gamma$ neighborhood $\left(W^{0}\right)_{0}$; it then follows that $p$ is a $\gamma$ continuous pseudo-norm and $\{x: p(x)<1\}$ is a $\gamma$ neighborhood of 0 . But then so also is $W$, since $W \supset\{x: p(x)<1\}$. This completes the proof.

Theorem 10. Let $(X, \mathcal{T})$ be an l.t.s., let the topology for $X^{*}$ of uniform convergence on $\mathcal{T}_{c}$ totally bounded sets be denoted by $\mathcal{T}_{c}$-u.c.t.b., and let $\mathrm{S}=S(X)$. Then: (1) $\mathcal{T}_{c}$-u.c.t.b. and $\left(S-w^{*}\right)_{c}$ are, respectively, the finest u.c. and finest locally convex topologies which coincide with $w^{*}$ on each equicontinuous subset of $X^{*}$; hence, $\mathcal{\mho}_{c}$-u.c.t.b. is coarser than $\left(\mathcal{S}-w^{*}\right)_{0}$ and $\left(\mathcal{S}-w^{*}\right)_{c}$ is coarser than $e-w^{*}$; (2) each $w^{*}$ closed neighborhood of 0 for $\left(\mathcal{S}-w^{*}\right)_{c}$ is a $\mathcal{G}_{c}$-u.c.t.b. neighborhood of 0 ; and (3) in order that $(X, \mathcal{\top})$ be pseudo-complete the following two conditions are together necessary and sufficient: (i) $\mathcal{T}_{c}$-u.c.t.b. $=\left(\mathcal{S}-w^{*}\right)_{c}$ and (ii) each $\mathcal{T}_{c}$ closed and $\mathcal{T}_{c}$ totally bounded set is $\mathcal{T}_{c}$ compact.

Proof. (1) Note that Theorems 6 and 7 together with Lemma 3 show that $\mathcal{T}_{c}$ is a locally convex linear topology for which $\left(X, \mathcal{T}_{c}\right)^{*}=(X, \mathcal{T})^{*}$ and $S\left(X, \mathcal{T}_{c}\right)=S(X, \mathcal{T})$. The statements of (1) concerning $\left(S-\mathrm{w}^{*}\right)_{0}$ then follow immediately from Theorem 8 , and those concerning $\mathcal{T}_{c}$-u.c.t.b. are an easy corollary of Lemma $5,(1)$ and of Theorem 9 if one takes the $\mathcal{T}$ of the lemma to be $\mathcal{T}_{c}$, the $V$ of Theorem 9 to be a local base for $\mathcal{T}_{c}$, and $G$ to be all finite subsets of $X$.

(2) Let $\mathcal{C}=\left\{N_{0}: N\right.$ is a convex symmetric $\mathrm{w}^{*}$ closed $\left(\mathcal{S}-\mathrm{w}^{*}\right)_{c}$ neighbor- 
hood of 0$\}$; this is an admissible family and, by definition, $C \in \mathcal{C}$ implies there exists $N$ a convex symmetric w* closed $\left(S-\mathrm{w}^{*}\right)_{c}$ neighborhood of 0 such that $C=N_{0}$. But then $C^{0}=\left(N_{0}\right)^{0}=N$; hence u.c. $\mathcal{C}$ has the family of all w* closed convex symmetric $\left(S-\mathrm{w}^{*}\right)_{c}$ neighborhoods of 0 as a local base and this is obviously coarser than the local base for $\left(\delta-w^{*}\right)_{c}$. Since the latter is coarser on each $S \in \mathcal{S}$ than is $\mathrm{w}^{*}=$ u.c. $G$ then so also is u.c. $\mathcal{C}$. Now apply Theorem 9 to obtain $\mathcal{C} \subset \mathcal{B}$, where here $\mathbb{B}$ is the family of all $\mathcal{G}_{c}$ totally bounded subsets of $X$; thus $N$ a w* closed convex and symmetric (S-w*)c neighborhood of 0 implies $N_{0}$ is $\mathcal{T}_{c}$ totally bounded and therefore $N=\left(N_{0}\right)^{0}$ is a $\mathcal{T}_{c}$-u.c.t.b. neighborhood of 0 .

(3) Suppose first that $X$ is pseudo-complete. Theorem 2 then shows that $\left(X, \mathcal{T}_{c}\right)$ is complete; hence each $\mathcal{T}_{c}$ closed and $\mathcal{T}_{c}$ totally bounded set is $\mathcal{T}_{c}$ compact and the Mackey-Arens Theorem [2] implies that $\left(X^{*}, \mathcal{\mho}_{c}\right.$-u.c.t.b.)* $=\left(X^{*}, \mathrm{w}^{*}\right)^{*}$. Now let $W$ be any $\left(\mathcal{S}-\mathrm{w}^{*}\right)_{c}$ neighborhood of 0 (where $W$ can be taken convex and symmetric); Part (2) of this theorem then implies that $\left(W_{0}\right)^{0}$ is a $\mathcal{T}_{c}$-u.c.t.b. neighborhood of 0 . If $\phi$ is a linear functional on $X^{*}$ such that $\phi(W)$ is bounded, then $\phi \in\left(X^{*},\left(\mathcal{S}-\mathrm{w}^{*}\right)_{c}\right)^{*}$, where the latter space is $\left(X^{*}, \mathrm{e}-\mathrm{w}^{*}\right)^{*}$ by Theorems 7 and 6 . But $X$ is, by hypothesis, pseudo-complete, and therefore $\phi$ is $\mathrm{w}^{*}$ continuous by Theorem 1 ; hence, $\phi$ is $\tau_{c}$-u.c.t.b. continuous. Lemma 9 then applies to show $W$ is a $\mathcal{T}_{c}$-u.c.t.b. neighborhood of 0 . To prove the converse of (3) it clearly suffices by Theorems 1,7 , and 6 to show that each $\left(\mathcal{S}-\mathrm{w}^{*}\right)_{c}$ continuous linear functional is $\mathrm{w}^{*}$ continuous. But this is clear by the hypotheses and by the Mackey-Arens Theorem [2]. This completes the proof.

REMARK. G. T. Roberts has recently established in [25] the fact that $\mathcal{T}_{c}$-u.c.t.b. is the finest u.c. topology coinciding with $\mathrm{w}^{*}$ on equicontinuous sets, but in a considerably less direct fashion. He is concerned there only with the finest u.c. topology and does not touch upon the remainder of our theorem.

The following two examples show that, in general, each of the first two topologies of $\mathcal{T}_{c}$-u.c.t.b., $\left(S-\mathrm{w}^{*}\right)_{c}$, and $\mathrm{e}-\mathrm{w}^{*}$ is properly coarser than the succeeding topology (each is coarser by the above theorem).

First, let the space $X$ in the above theorem be the adjoint $E^{*}$ of an infinite-dimensional Banach space $E$, supplied with the ${ }^{*}$ topology of $E^{*}$. Since this topology is locally convex, the topology $\mathcal{G}_{c}$ is simply $\mathrm{w}^{*}$ itself. Further, it is clear that $X^{*}=E$ and the topology $\mathcal{\sigma}_{c}$-u.c.t.b. is simply the norm topology of $E$. In $E^{*}$ each $\mathrm{w}^{*}$ bounded set is equicontinuous on $E$ [3]; hence each $\mathcal{T}_{c}$ closed and totally bounded set is $\mathcal{T}_{c}$ compact. It then follows from Theorem 10 that if the finest u.c. and finest locally convex topologies coincide (each finest with respect to the property that each coincides with $\mathrm{w}^{*}$ on equicontinuous subsets of $\left.X^{*}\right), X$ is pseudo-complete. However, it is well known that here $X$ is not complete and therefore is not pseudo-complete.

A modification of the above example is used now to show that $\left(\mathcal{S}-\mathrm{w}^{*}\right)_{c}$ is, 
in general, properly coarser than e-w*. Let $E$ be the space of the previous example and let $\mathcal{T}_{c}$ be the finest locally convex linear topology for $E$. Kaplan has shown [14] that the space $X=\left(E, \mathcal{V}_{c}\right)$ is a complete 1.t.s. and it is also known (e.g., see [14]) that every linear functional on $X$ is continuous. Thus, the adjoint space $X^{*}$ is simply the space of all linear functionals on $E$ while, as is well known, the adjoint space $E^{*}$ (=all norm continuous linear functionals on $E$ ) is dense in $X^{*}$ with respect to the $w^{*}$ topology of $X^{*}$. However, as was noticed in the previous example, $E^{*}$ is a proper linear subspace of $X^{*}$; hence, $E^{*}$ is not $\mathrm{w}^{*}$ closed in $X^{*}$. Now if $S \in S(X)$, there exists some convex radial neighborhood of $0 U \subset X$ such that $S$ is the polar (in $X^{*}$ ) of $U$. The polar $U^{0}$ of $U$ in $E^{*}$ is then $\mathrm{w}^{*}$ bounded in $E^{*}$, hence is equicontinuous on $E$ (the latter since $E$ is a Banach space [3]). But then $E^{*} \cap S=E^{*} \cap U^{0}=U^{0}$, and the latter is a $\mathrm{w}^{*}$ compact subset of $E^{*}$; hence is a $\mathrm{w}^{*}$ closed subset of $X^{*}$. Thus, $E^{*}$ is an e-w* closed linear subspace of $X^{*}$ which is not w* closed. Since the space $\left(X^{*}, \mathcal{T}_{c} \text {-u.c.t.b.) }\right)^{*}$ is $X$ and $E^{*}$ is a convex subset of $X^{*}$ it is then clear by the Mackey-Arens theorem [2] that $E^{*}$ cannot be $\mathcal{T}_{c}$-u.c.t.b. closed. However, Theorem 10 implies that $\mathcal{T}_{c}$-u.c.t.b. $=\left(\mathcal{S}-\mathrm{w}^{*}\right)_{c}$, and thus $E^{*}$ is $\mathrm{e}-\mathrm{w}^{*}$ closed but not $\left(\mathcal{S}-\mathrm{w}^{*}\right)_{c}$ closed; hence $\left(\mathcal{S}-\mathrm{w}^{*}\right)_{c}$ is properly coarser than e-w*.

The results of these two examples are summarized in the following

Theorem 11. Let $X$ be an l.t.s. Then each of these topologies for $X^{*}$ is, in general, properly coarser than the succeeding topology: the finest u.c., the finest locally convex, and the finest, where each is finest with respect to the property "each coincides with w* on each equicontinuous subset of $X^{*}$."

The remainder of this section is given over to determining a sufficient condition that these three topologies shall coincide. We should remark that the first statement of the following theorem is an extension and clarification of a result already known for $F$ spaces [6, Theorem 5]. We utilize a lemma which, except for minor modifications in statement and proof, was proved in the paper quoted above. The proof is omitted.

Lemma 10. Let $X$ be a pseudo-metrisable l.t.s., let $W$ be an open e-w* neighborhood of 0 in $X^{*}$, and suppose $\left\{U_{n}\right\}$ is a monotone decreasing sequence of neighborhoods forming a local base for $X$. Then there exists a finite set $A_{1} \subset U_{1}$ and, for every $n \geqq 1$, there exists a finite set $B_{n} \subset U_{n}$ such that, if $A_{n+1}=A_{n} \cup B_{n}$, then $\left(A_{n}\right)^{0} \cap\left(U_{n}\right)^{\circ} \subset W$.

Theorem 12. Let $(X, \mathcal{T})$ be a pseudo-metrisable l.t.s. and denote by u.c.t.b. the topology for $(X, \mathcal{T})^{*}$ of uniform convergence on all $\mathcal{T}$ totally bounded subsets of $X$. Then, the topologies u.c.t.b. and e-w* coincide; hence these three topologies coincide: the finest u.c. topology, the finest locally convex topology, and the finest topology, each being finest with respect to coinciding with w* on each equicontinuous set of $(X, \mathcal{T})^{*}$. 
Proof. Note that, since $\mathcal{T}_{c}\left(\mathcal{T}_{c}\right.$ as in $\left.\S 1\right)$ is coarser than $\mathcal{T}$, each $\mathcal{T}$ totally bounded set is $\mathcal{T}_{c}$ totally bounded; hence u.c.t.b. is coarser than $\mathcal{T}_{c}$-u.c.t.b. The entire theorem will thus (by Theorem 10) be proved if it can be shown that e-w* is coarser than u.c.t.b. Again, since it is clear by Theorem $\mathbf{5}$ that $\mathrm{e}-\mathrm{w}^{*}$ is a semi-linear topology it is sufficient to show each e-w* open neighborhood $W$ of 0 contains the polar $A^{0}$ of some $\mathcal{T}$ totally bounded set $A$. Using Lemma 10, if $\left\{U_{n}\right\},\left\{B_{n}\right\}$, and $\left\{A_{n}\right\}$ are as in that lemma, we set $A=\cup_{n=1}^{\infty} A_{n}$ and show: (1) $A$ is $\mathcal{T}$ totally bounded; (2) $A^{\circ} \subset W$. To prove (1), let $U$ be a $\mathcal{T}$ neighborhood of 0 . By the monotoneity of the local base $\left\{U_{n}\right\}$ there is a positive integer $N$ for which $n \geqq N$ implies $B_{n} \subset U_{n} \subset U_{N} \subset U$. Let $B=\bigcup_{p<N}\left(B_{p} \cup\{0\}\right)$. In Lemma 10, $A_{1}=\{0\}$; hence $A \subset \bigcup_{n \geqq N}\left(B_{n} \cup B\right)$ $\subset U_{N} \cup B \subset U+B$, and $A$ is $\mathcal{T}$ totally bounded. We now demonstrate (2). By definition of $A$ it is clear that $A^{0} C\left(A_{n}\right)^{0}$ for each integer $n$, and therefore $A^{0} \cap\left(U_{n}\right)^{0} \subset\left(A_{n}\right)^{0} \cap\left(U_{n}\right)^{0} \subset W$. But then $A^{0}=A^{0} \cap X^{*}=A^{0} \cap \bigcup_{n=1}^{\infty} \quad\left(U_{n}\right)^{0}$ $=\bigcup_{n=1}^{\infty}\left(A^{0} \cap\left(U_{n}\right)^{0}\right) \subset W$, and the theorem is concluded.

\section{Part 3. Fully complete spaces}

8. As pointed out previously in $\$ 3$, our initial interest in pseudo-completeness was aroused by the role it seemed to play in weak compactness (e.g., see [8]). Going even further back, certain concepts and theorems of which our pseudo-completeness and theorems relating pseudo-completeness to completeness represent partial generalizations occupy an impressive place in the literature. Implicit in the Banach development [3, pp. 131-132] is the theorem: if $X$ is a Banach space and $\phi$ a linear functional on $X^{*}$ such that $K_{\phi} \cap S$ is w* closed ( $S$ is the norm closed unit sphere of $X^{*}$ ), then $K_{\phi}$ is $\mathrm{w}^{*}$ closed. Later, first Alaoglu [1], then Krein and Smulian [19] proved the above theorem for arbitrary linear subspaces of $X^{*}$. Somewhat after this Katětov [15] showed that a linear normed space $X$ is complete if and only if each linear subspace $L$ of $X^{*}$ is $\mathrm{w}^{*}$ closed whenever $L \cap S$ is w* closed. It is to Munroe [22] that we owe the term "bounded-weak" (from which our "equicontinuous- $w^{*}$ " is derived), and certain conjectural generalizations of the above theorem are readily stated in terms of the topology e-w*. For example, the fact that in a linear normed space $X$ a linear subspace of $X^{*}$ intersects the closed unit sphere in a $\mathrm{w}^{*}$ closed set if and only if it intersects each $S \in S(X)$ in a $\mathrm{w}^{*}$ closed set suggests the following generalization of the Katětov theorem: an 1.t.s. $X$ is complete if and only if each e-w* closed linear subspace of $X^{*}$ is also $\mathrm{w}^{*}$ closed. This statement is false, even for locally convex 1.t.s.'s, as we show by an example following Theorem 14 . We do give an extension of the Katětov theorem in Corollary 16.1. Note also that our Theorem 2 gives a weakened (in one direction) version of the Katětov result stated for arbitrary 1.t.s.'s. A natural question is this: for what type 1.t.s. is the Katětov result still true? We show in Corollary 16.1 (part of this result was shown in [6]) that our rephrasing of the Katětov theorem holds for 
pseudo-metrisable 1.t.s.'s and in Corollary 17.2 that it holds also for a certain additional class of spaces. A complete answer is not given here. However, the property exhibited in the Katětov, Alaoglu, and Krein-Smulian theorems is of sufficient interest, we feel, to merit an independent study. This is the purpose which motivates the remainder of this paper.

If $X$ is an 1.t.s., recall that $X$ is fully complete if a linear subspace (arbitrary) of $X^{*}$ is $\mathrm{w}^{*}$ closed if and only if it is e-w* closed. Note that full completeness obviously implies pseudo-completeness and also implies completeness for locally convex spaces, by our Theorem 2. We shall show in Corollary 14.1 that it is, in general, stronger than both completeness and pseudocompleteness. Preliminary to these and other results we need a lemma, which we state as Corollary 13.2. This result is well known [7, Theorem $8 ; 19$, Theorem 12'] but the construction used in our proof is needed later, as well as the corollary itself.

THEOREM 13. Let $G_{1}, G_{2}$, and $H$ be groups (no topology is implied), with $H$ abelian, and let $h$ be a homomorphism of $G_{1}$ onto $G_{2}$. Let $H\left(G_{i}, H\right)$ be the set of all homomorphisms of $G_{i}$ into $H(i=1,2)$, and let $\Gamma$ be the subset of $H\left(G_{1}, H\right)$ consisting of all $f$ such that $K_{h} \subset K_{f}$. There is then an isomorphism $J$ of $\Gamma$ onto $H\left(G_{2}, H\right)$. When $G_{1}, G_{2}$, and $H$ are topological groups and $h$ is a continuous-open homomorphism of $G_{1}$ onto $G_{2}$, let $C\left(G_{i}, H\right)$ denote the set of all continuous homomorphisms of $G_{i}$ into $H(i=1,2)$ and let $T$ be $J$ restricted to $\Gamma \cap C\left(G_{1}, H\right)$. Then $T$ is an algebraic isomorphism of $\Gamma \cap C\left(G_{1}, H\right)$ onto $C\left(G_{2}, H\right)$.

Proof. The proof is a well known construction and depends essentially on the induced homomorphism theorem [9]. For the sake of later work, however, we write down $J$ explicitly. For any element $f$ of $H\left(G_{1}, H\right)$ we obtain by that theorem a $g$ in $H\left(G_{2}, H\right)$ which is unique with respect to the relation: $g(h)=f$. We then set $J(f)=g$.

When $C\left(G_{i}, H\right)$ is given various topologies we can discuss the continuity properties of $J$. If $\mathscr{D}_{i}(i=1,2)$ is a family of subsets of $G_{i}$ such that $A, B \in \mathscr{D}_{i}$ implies there exists $C \in \mathscr{D}_{i}$ such that $A \cup B \subset C$, we take as a base for neighborhoods of 0 in the u.c. $D_{i}$ topology of $C\left(G_{i}, H\right)$ all sets of the form $(D, U)$, where $D \in \Phi_{i}, U$ is a neighborhood of 0 in $H$, and $(D, U)$ is the set of all $F \in C\left(G_{i}, H\right)$ for which $F(D) \subset U$. We consider two conditions on $\mathscr{D}_{i}(i=1,2)$ : (a) $B \in \mathscr{D}_{2}$ implies there exists $A \in \mathscr{D}_{1}$ such that $B \subset h(A)$; (b) $A \in \mathscr{D}_{1}$ implies there exists $B \in \mathscr{D}_{2}$ such that $h(A) \subset B$.

The following easy corollary is stated for reference.

Corollary 13.1. As a function from $\left[\Gamma \cap C\left(G_{1}, H\right)\right.$, u.c. $\left.\mathcal{D}_{1}\right]$ to $\left[C\left(G_{2}, H\right)\right.$, u.c. $\left.\mathscr{D}_{2}\right] T$ is continuous if (a) holds and open if (b) holds.

The following two special cases of the previous corollary are stated as Corollary 13.2, and result upon taking $\mathscr{D}_{1}$ and $\mathscr{D}_{2}$ first to be each the family of finite sets and second to be each the family of all bounded sets of $G_{1}=X$ and $G_{2}=X / L$ respectively. 
Corollary 13.2. (1) Let $X$ be an l.t.s. and $L$ be a closed linear subspace of $X$. Then the polar $L^{0}$ of $L$ in $X^{*}$ and $(X / L)^{*}[X / L$ denotes the quotient space of $X$ by $L]$ are topologically isomorphic when each of these spaces is supplied with its $w^{*}$ topology; (2) let $X$ be an l.t.s. having the property that, for any closed linear subspace $L$ of $X$, the natural map $h$ of $X$ onto $X / L$ is such that $B$ bounded in $X / L$ implies there exists $A$ bounded in $X$ for which $B C h(A)$. Then the spaces $L^{0}$ and $(X / L)^{*}$ are topologically isomorphic when each is supplied with its topology of uniform convergence on bounded subsets (of $X$ and $X / L$ respectively).

REMARK. The condition on $h$ in (2) above holds in linear normed spaces [13]. This remark coupled with (2) above yields a result of Krein-Smulian [19]. Part (1) of Corollary 13.2 is a classical theorem due to Dieudonné [7]. We now discuss with the aid of Corollary 13.2 (and of the function $T$ defined in Theorem 13) the algebra of full completeness. Some few preliminaries are needed. If $X$ and $Y$ are 1.t.s.'s and $F$ is a continuous linear operator on $X$ to $Y$, the adjoint operator (to $F$ ) on $Y^{*}$ to $X^{*}$ will be denoted by $F^{*}$ (for $g \in Y^{*}$ and $x \in X, F^{*} g(x)=g(F(x))$ ), and is known to be well defined and continuous in various topologies for $Y^{*}$ and $X^{*}$. In the following theorems (particularly in Theorems 14 and 15) we present all the known properties of full completeness.

THEOREM 14. Let $X$ and $Y$ be l.t.s.'s, and let $h$ be a linear operator which is continous-open on $X$ onto $Y$. Then $X$ fully complete implies $Y$ fully complete.

Proof. Let $Z$ be an e-w* closed linear subspace of $Y^{*}$ and let $T$ be the isomorphism of $\Gamma^{\prime} \subset X^{*}$ onto $Y^{*}$ of Theorem 13, where $\Gamma^{\prime}=\left\{f: f \in X^{*}\right.$ and $\left.K_{h} \subset K_{f}\right\}$. Since $h$ is continuous and open, $T$ is bicontinuous on $\Gamma^{\prime}$ onto $Y^{*}$, where each has its $\mathrm{w}^{*}$ topology. Let $H=T^{+1}(Z)$. To prove $Z$ is $\mathrm{w}^{*}$ closed, it suffices to prove $H$ is w* closed in $Y^{*}$, for then $Z=T(H)$ is w* closed. Again, it is sufficient to prove that $H \cap U^{0}$ is $\mathrm{w}^{*}$ closed for every neighborhood $U$ of 0 in $X$, and to do this it is enough to prove $T\left(H \cap U^{0}\right)$ is $\mathrm{w}^{*}$ closed in $Y^{*}$; i.e., $Z \cap T\left(\Gamma^{\prime} \cap U^{0}\right)$ is w* closed. Let $V=h(U)$; then $V$ is a neighborhood of 0 in $Y$. If we can show that $T\left(\Gamma^{\prime} \cap U^{0}\right) \subset V^{0}$, then $Z \cap T\left(\Gamma^{\prime} \cap U^{0}\right)=\left(Z \cap V^{0}\right)$ $\cap T\left(\Gamma^{\prime} \cap U^{0}\right)$, where $Z \cap V^{0}$ is $\mathrm{w}^{*}$ closed since $Z$ is e-w* closed. But $T\left(\Gamma^{\prime} \cap U^{0}\right)$ is $\mathrm{w}^{*}$ compact (hence closed) since $U^{0}$ is $\mathrm{w}^{*}$ compact and $T$ is $\mathrm{w}^{*}$ continuous. Hence, it suffices to prove $T\left(\Gamma^{\prime} \cap U^{0}\right) \subset V^{0}$. For this purpose, let $f \in \Gamma^{\prime} \cap U^{0}$, and let $g=T(f)$. Then $|f(x)| \leqq 1$ for all $x \in U$, and $f(x)=g(h(x))$ for every $x \in X$. Hence $|g(y)| \leqq 1$ for every $y$ in $V=h(U)$; thus $g \in V^{0}$, and the proof is complete.

One of the most striking properties of full completeness is that given by the previous theorem. A particular corollary of Theorem 14 is of especial interest since it, in conjunction with some work of Kothe, can be used to show full completeness is stronger than completeness for locally convex spaces. More specifically, if $X$ is an 1.t.s. and $L$ is a linear subspace of $X$, then 
the quotient space $X / L$ (supplied with the quotient topology) is fully complete whenever $X$ is fully complete. On the other hand, Kothe has shown [18] that there exists a complete (hence pseudo-complete) locally convex space $X$ and a closed linear subspace $L$ of $X$ such that $X / L$ is not complete. It is thus apparent that full completeness is stronger than pseudo-completeness and is also stronger than completeness in locally convex spaces. A more direct example is obtained from the first example given following Theorem 10 of the previous section. There it is shown that there exists a complete locally convex 1.t.s. (Hausdorff) and an e-w* closed linear subspace of its adjoint which is not $\mathrm{w}^{*}$ closed; hence completeness does not imply full completeness. These results are summarized in the following corollary.

Corollary 14.1. The quotient space $X / L$ of an l.t.s. $X$ by a linear subspace $L$ of $X$ is fully complete if $X$ is fully complete. Full completeness is stronger than pseudo-completeness and is stronger than completeness for locally convex spaces.

A theorem which is dual to Theorem 14 is obtained next.

THEOREM 15. If $h$ is a linear continuous operator on $X$ to $Y$, where $X$ and $Y$ are l.t.s.'s, if $h^{*}$, the adjoint operator of $h$, is $w^{*}$ open on $Y^{*}$ onto $X^{*}$, and if $Y$ is fully complete, then so also is $X$ fully complete.

Proof. Since $h^{*}$ is, by hypothesis, $\mathrm{w}^{*}$ open on $Y^{*}$ onto $X^{*}$, it is $\mathrm{w}^{*}$ continuous and open. It is then clear that there is induced a topological isomorphism $F$ on $\left(Y^{*} / K_{h^{*}}, \mathrm{w}^{*}\right)$ onto $\left(X^{*}, \mathrm{w}^{*}\right)$ such that $F(p)=h^{*}$, where $p$ is the natural mapping of $Y^{*}$ onto $Y^{*} / K_{h^{*}}$. Now, let $Z$ be an e-w* closed linear subspace of $X^{*}$ and let $H$ be the inverse of $Z$ under $h^{*}$. The latter is $p^{-1}\left(F^{-1}(Z)\right)$. It is sufficient to show that $H$ is e-w* closed, for then $H$ is w* closed and hence (since $p$ is a natural map) $p(H)=F^{-1}(Z)$ is $\mathrm{w}^{*}$ closed. But then $F\left(F^{-1}(Z)\right)=Z$ is w $^{*}$ closed since $F$ is topological. We show then, if $U$ is an arbitrary neighborhood of 0 in $Y$, that $H \cap U^{0}$ is $\mathrm{w}^{*}$ closed. Observe that $V=h^{-1}(U)$ is a neighborhood of 0 in $X$, and, by assumption, that $Z \cap V^{0}$ is $\mathrm{w}^{*}$ closed. Hence $h^{*-1}\left(Z \cap V^{0}\right)=H \cap h^{*-1}\left(V^{0}\right)$ is $\mathrm{w}^{*}$ closed. However, $h^{*-1}\left(V^{0}\right)=h(V)^{0}$ and thus $H \cap h(V)^{0}$ is $\mathrm{w}^{*}$ closed. Since $h(V) \subset U$, we have $U^{0} C h(V)^{0}$; hence $H \cap U^{0}=H \cap h(V)^{0} \cap U^{0}=\left[H \cap h(V)^{0}\right] \cap U^{0}$, and this is w* closed. The proof is thus completed.

The next two corollaries present properties of full completeness which have similiar completeness analogues, and are obtained by taking first $Y$ to be an 1.t.s., $L \subset Y$ a closed linear subspace to be the $X$ of the above theorem, and by taking the $h$ of the theorem to be the identity function on $L$ into $Y$ ( $h^{*}$ is $\mathrm{w}^{*}$ onto and open by a theorem of Dieudonné [7]); secondly, by letting $E$ be a linear space with two linear topologies $t$ and $\mathcal{T}$ giving the same adjoint and such that $(E, \mathcal{T})$ is fully complete and $t$ is finer than $\mathcal{T}$. Here we let $(E, t),(E, \mathcal{T})$, and the identity function on $(E, t)$ onto $(E, \mathcal{T})$ be respectively the $X, Y$, and $h$ of the above theorem. 
Corollary 15.1. Let $Y$ be a locally convex l.t.s., $L \subset Y$ be a closed linear subspace, and suppose $Y$ is fully complete. Then $L$ is also fully complete with respect to its relative topology.

Corollary 15.2. Let $E$ be a linear space with $t$ and $\mathcal{T}$ two topologies for $E$ which are linear and which give the same adjoint. Suppose further that $(E, \mathcal{T})$ is fully complete and $t$ is finer than $\mathcal{T}$. Then $(E, t)$ is also fully complete.

We exhibit in the final two paragraphs below two classes of spaces which are fully complete (Theorem 16 and Corollary 17.2) and indicate some types of spaces which are not fully complete. The following theorem shows that a pseudo-metrisable space which is pseudo-complete is also fully complete (in fact, a stronger result is obtained), and a later theorem shows that an arbitrary cartesian product of reals is always fully complete.

Theorem 16. Let $(X, \mathcal{T})$ be a pseudo-metrisable l.t.s. These conditions are then equivalent: (a) $(X, \mathcal{T})$ is pseudo-complete; (b) $(X, \mathcal{T})$ is fully complete; and (3) a convex subset $C$ of $(X, \mathcal{T})^{*}$ is $w^{*}$ closed if and only if it is $e-w^{*}$ closed; i.e., $C$ is w* closed if and only if $S \in S(X)$ implies $C \cap S$ is w* closed.

Proof. It clearly suffices to prove each e-w* closed and convex set in $X^{*}$ is $\mathrm{w}^{*}$ closed when $X$ is pseudo-complete, so suppose $X$ is pseudo-complete, and let $C \subset X^{*}$ be any convex set which is e-w* closed. By Theorem $12, C$ is $\mathcal{T}_{c}$-u.c.t.b. closed. But then, since $X$ is pseudo-complete by hypothesis, every $\mathcal{T}_{c}$ totally bounded and closed set in $X$ is $\mathcal{T}_{c}$ compact; the Mackey-Arens theorem then implies that $\left(X^{*}, \mathcal{\sigma}_{c} \text {-u.c.t.b. }\right)^{*}=X$. The latter fact implies that $C$ is also $w^{*}$ closed, it being $\mathcal{T}_{c}$-u.c.t.b. closed and convex. This concludes the proof.

The following corollary is stated primarily to relate the above theorem to the more familiar concept of completeness. Its proof is easily made in virtue of the above theorem and of Theorem 2. Part (c) below is, we feel, a particularly interesting characterization of completeness for locally convex pseudometrisable spaces.

Corollary 16.1. When $X$ is a locally convex and pseudo-metrisable l.t.s. these conditions are equivalent: (a) $X$ is complete; (b) $X$ is fully complete; and (c) a convex subset $C$ of $X^{*}$ is $w^{*}$ closed if and only if $C \cap S$ is $w^{*}$ closed for every $S \in S(X)$.

REMARK. The implication (a) $\rightarrow$ (b) of the corollary was obtained, for linear normed spaces, by Alaoglu [1] and by Krein-Smulian [19], and for metrisable locally convex spaces by Dieudonné and Schwartz [6]. In addition, Katětov established, again for linear normed spaces, the equivalence of (a) and (b) in [15]. Finally, Ptak has quite recently in [24] duplicated some of our results concerning pseudo-completeness and full completeness. For example, he has proved (for locally convex spaces) our Lemma 2 (actually, only $(1) \leftrightarrow(3)$ of that lemma), Theorem 2 , and $(a) \leftrightarrow(b)$ of Theorem 16 for 
metrisable spaces. In addition, he has obtained this characterization of fully complete spaces: a locally convex space $X$ is fully complete if and only if every linear continuous operator of $X$ onto a locally convex space which takes open sets into somewhere dense sets is already open. Ptak does not exhibit any of the other properties of full completeness; nor does he touch upon any of our results concerning the topology e-w*.

The corollary above can be used to show that full completeness is not preserved under the operation of taking arbitrary cartesian products. In fact, let $X$ be any locally convex Hausdorff l.t.s. which is complete but not fully complete (such spaces exist by our examples following Theorem 14). As is well known [21, Proposition 2.7] $X$ is topologically isomorphic with a closed linear subspace of a cartesian product $\mathrm{P} X_{a}$, where each $X_{a}$ is a Banach space. If $\mathrm{P} X_{a}$ were fully complete (here each $X_{a}$ is fully complete by Corollary 16.1) so also would $L$ be fully complete by Theorem 14 and Corollary 15.1 , contradicting the fact that $L$ is not fully complete $\left({ }^{2}\right)$.

A rather curious property of full completeness, in virtue of the preceding example, can be stated concerning arbitrary cartesian products $\mathrm{P} X_{a}$, where each $X_{a}$ is a copy of the real numbers. Such spaces $X=\mathrm{P} X_{a}$ (P $X_{a}$ has its cartesian product topology) have been labeled entire spaces by Kaplan [14]. The theorem that such spaces are always fully complete will be drawn as a corollary (Corollary 17.2) of the following theorem.

THEOREM 17. Let $X$ be an l.t.s. such that $X^{*}$ is total for $X$, and let $E$ be the space $\left(X^{*}, w^{*}\right)$. Then, every linear subspace $L$ of $E^{*}$ is e-w* closed; i.e., $L \cap S$ is weakly closed in $X$ for every $S \in S(E)$.

Proof. Here we regard $X$ as the adjoint of $E$ and observe that $S \in S(E)$ if and only if $S \subset X$ is weakly closed, convex, symmetric, and equicontinuous on $E=\left(X^{*}, \mathrm{w}^{*}\right)$; it follows easily that $S(E)$ is the family of all weakly closed convex and symmetric hulls of finite sets of $X$. Since this is so and since the weak topology of $X$ is Hausdorff, then each $S \in S(E)$ is contained in the linear subspace $L(M)$ spanned by some finite set $M$ (each such finite subspace being weakly closed [29]). But then if $L$ is a linear subspace of $E^{*}$ and $S \in S(E)$, we have $L \cap S=L \cap(S \cap L(M))=L \cap L(M) \cap S$, where $L \cap L(M)$ and $S$ are weakly closed; hence $L \cap S$ is weakly closed.

Corollary 17.1. Let $X$ be an l.t.s. such that $X^{*}$ is total for $X$ and let $E=\left(X^{*}, w^{*}\right)$. Then, in $E$, full completeness and pseudo-completeness are equivalent, each being equivalent to the condition: every linear subspace of $X$ is weakly closed.

Proof. It is clearly sufficient to show that pseudo-completeness implies the condition. By the theorem and the assumption of pseudo-completeness, every

(2) This example was suggested to the author by E. Michael. 
maximal proper linear subspace of $X$ is weakly closed. But then, so is every linear subspace of $X$, since any linear subspace can be written as an intersection of maximal proper linear subspaces.

REMARK. The duals of Theorem 17 and Corollary 17.1 (obtained by interchanging $X^{*}$ and $X, \mathrm{w}^{*}$ and weak) are also valid, and yield the statement: if $X$ is an 1.t.s. and $E=\left(X\right.$, weak), then in $E^{*}$ every linear subspace is e-w* closed; i.e., $L \subset X^{*}$ a linear subspace implies $L \cap S$ is $\mathrm{w}^{*}$ closed for every $S \in S(E)$. In particular, in $E$ pseudo-completeness and full completeness are equivalent, each being equivalent to the condition: every linear subspace of $E^{*}=X^{*}$ is $\mathrm{w}^{*}$ closed.

COROLlARY 17.2. Let $E$ be an arbitrary cartesian product of copies of the real numbers, supplied with the cartesian product topology. Then $E$ is fully complete.

Proof. It is known [14] that the topology of $E$ is its weak topology. Since $E$ is complete, it is also pseudo-complete by Theorem 2 . But then the remark preceding the corollary shows that $E$ is then fully complete.

Direct (topological) sums of fully complete spaces are not fully complete, as can be seen by the following example. If $\left\{X_{a}: a \in A\right\}$ is a family of locally convex 1.t.s.'s, we take as a local base for the weak product of the $X_{a}$ 's all convex and symmetric subsets $W$ such that $W \cap X_{a}$ is a neighborhood of 0 in $X_{a}$ for every $a \in A$ (here $X_{a}$ is identified with its natural image in the weak product space). The weak product with this topology is denoted by $\sum_{a \in A} X_{a}$ and is known to be a locally convex l.t.s. [4]. Now, let $B$ be any infinitedimensional Banach space and let $\gamma$ be the finest locally convex linear topology for $B$. It is known that $(B, \gamma)$ is nothing more than $\sum_{a \in A} X_{a}$, where $A$ is a Hamel base for $B$ and each $X_{a}$ is a copy of the reals. However, we have already seen in the example preceding Corollary 14.1 that $(B, \gamma)$ is not fully complete, although each $X_{a}$ is fully complete by Corollary 16.1 .

\section{BIBLIOGRAPHY}

1. Leonidas Alaoglu, Weak topologies of normed linear spaces, Ann. of Math. vol. 41 (1940) pp. 252-267.

2. R. F. Arens, Duality in linear spaces, Duke Math. J. vol. 14 (1947) pp. 787-794.

3. Stefan Banach, Theorie des opérations linéaires, Warsaw, 1932.

4. N. Bourbaki, Espaces vectoriel topologiques, Actualités Scientifiques et Industriells, no. 1189, Paris, Hermann, 1953.

5. J. Dieudonné, Natural homomorphisms in Banach spaces, Proc. Amer. Math. Soc. vol. 1 (1950) pp. 54-59.

6. J. Dieudonné and L. Schwartz, La dualité dans les espaces $F$ et $L F$, Annales de L'Institut Fourier vol. 1 (1949) pp. 61-101.

7. J. Dieudonné, La dualité dans les espaces vectoriels topologiques, Annales de L'École Normale Superieure vol. 59 (1942) pp. 107-139.

8. W. F. Eberlein, Weak compactness in Banach spaces, Proc. Nat. Acad. Sci. U.S.A. vol. 33 (1947) pp. 51-53. 
9. S. Eilenberg and C. Kuratowski, Théoremes d'addition, Fund. Math. vol. 35 (1939) pp. 193-200.

10. David Gale, Compact sets of functions and function rings, Proc. Amer. Math. Soc. vol. 1 (1950) pp. 303-308.

11. A. Grothendieck, Critêres de compacité dans les espaces fonctionnels généraux, Amer. J. Math. vol. 74 (1952) pp. 168-188.

12. - Sur la complétion du dual d'un espace vectoriel localement convexe, C. R. Acad. Sci. Paris vol. 230 (1950) pp. 605-606.

13. Felix Hausdorff, Théorie der linearen metrischen Räume, J. Reine Angew. Math. vol. 167 (1932) pp. 294-311.

14. Samuel Kaplan, Cartesian products of reals, Amer. J. Math. vol. 74 (1952).

15. M. Katětov, Über normierte Vektorräume, Bull. Int. Acad. Tcheque vols. 43-44 (1942-1943).

16. J. L. Kelley, Convergence in topology, Duke Math. J. vol. 17 (1950) pp. 277-283.

17. V. L. Klee, Convex sets in linear spaces, Duke Math. J. vol. 18 (1951) pp. 443-466.

18. G. Kothe, Die Quotientenräume eines linearen vollkommenen Räumes, Math. Zeit. vol. 51 (1947) pp. 17-35.

19. M. Krein and V. Smulian, On regularly convex sets in the space conjugate to a Banach space, Ann. of Math. vol. 41 (1940) pp. 556-583.

20. G. W. Mackey, On infinite dimensional linear spaces, Trans. Amer. Math. Soc. vol. 57 (1945) pp. 153-207.

21. E. A. Michael, Locally multiplicatively-convex topological algebras, Memoirs of the American Mathematical Society, no. 11, 1952.

22. M. E. Munroe, Homomorphisms on Banach spaces, Bull. Amer. Math. Soc. vol. 54 (1948) pp. 776-781.

23. R. S. Phillips, On weakly compact subsets of a Banach space, Amer. J. Math. vol. 65 (1943) pp. 108-136.

24. Vlastimil Ptak, On complete topological linear spaces, Cehoslovack. Mat. Ž. (1953) pp. 301-364.

25. G. T. Roberts, The bounded-weak topology and completeness in vector spaces, Proc. Cambridge Philos. Soc. vol. 49 (1953) pp. 183-189.

26. V. Śmulian, Sur les ensembles régulierement fermés et faiblement compacts dans les espaces du type (B), C. R. (Doklady) Acad. Sci. URSS vol. 18 (1938) pp. 405-407.

27. — - Über lineare topologische Räume, Mat. Sbornik N.S. vol. 7 (1941) pp. 425-448.

28. A. Tychonoff, Über einen Funktionräum, Math. Ann. vol. 111 (1934) pp. 762-766.

29. - Ein Fixpunktsatz, Math. Ann. vol. 111 (1935) pp. 767-776.

30. John von Neumann, On complete topological spaces, Trans. Amer. Math. Soc. vol. 37 (1935) pp. 1-20.

TUlane University, New Orleans, La. 